\title{
Global Existence and Large Time Asymptotic Behavior of Strong Solution to the Cauchy Problem of 2D Density-Dependent Boussinesq Equations of Korteweg Type
}

\author{
Qi Zhang \\ University of Shanghai for Science and Technology, Shanghai, China \\ Email: zhangqi_8694@163.com
}

How to cite this paper: Zhang, Q. (2021) Global Existence and Large Time Asymptotic Behavior of Strong Solution to the Cauchy Problem of 2D Density-Dependent Boussinesq Equations of Korteweg Type. Advances in Pure Mathematics, 11, 346-368. https://doi.org/10.4236/apm.2021.114022

Received: March 22, 2021

Accepted: April 26, 2021

Published: April 29, 2021

Copyright ( 2021 by author(s) and Scientific Research Publishing Inc. This work is licensed under the Creative Commons Attribution International License (CC BY 4.0).

http://creativecommons.org/licenses/by/4.0/

\section{(c) (i) Open Access}

\begin{abstract}
In this paper, we study the Cauchy problem of the density-dependent Boussinesq equations of Korteweg type on the whole space with a vacuum. It is proved that there exists a unique strong solution for the two-dimensional Cauchy problem established that the initial density and the initial temperature decay not extremely slow. Particularly, it is allowed to be arbitrarily large for the initial data and vacuum states for the initial density, even including the compact support. Moreover, when the density depends on the Korteweg term with the viscosity coefficient and capillary coefficient, we obtain a consistent priority estimate by the energy method, and extend the local strong solutions to the global strong solutions. Finally, when the pressure and external force are not affected, we deform the fluid models of Korteweg type, we can obtain the large time decay rates of the gradients of velocity, temperature and pressure.
\end{abstract}

\section{Keywords}

Incompressible Boussinesq Equation, Korteweg Type, Global Strong Solutions, Large Time Behavior, Vacuum

\section{Introduction}

In this paper, we consider the system for the nonhomogeneous incompressible Boussinesq equations of Korteweg type as follows

$$
\left\{\begin{array}{l}
\rho_{t}+\operatorname{div}(\rho u)=0 \\
(\rho u)_{t}+\operatorname{div}(\rho u \otimes u)+\nabla p=\mu \Delta u+v \rho \nabla \Delta \rho+f, \quad x=\left(x_{1}, x_{2}\right) \in \Omega, t \geq 0 \\
\theta_{t}+u \cdot \nabla \theta-\kappa \Delta \theta=0 \\
\operatorname{div} u=0
\end{array}\right.
$$


Among them, the free vector field of divergence $u=\left(u^{1}, u^{2}\right)(x, t)$ represents the velocity of the fluid; the scalar function $p=p(x, t)$ and $\theta=\theta(x, t)$ represent pressure of the fluid and temperature respectively; parameter $\mu>0$ represents the viscosity coefficient dependent on temperature; $\rho=\rho(x, t)$ and $v$ represent density and the capillary coefficient respectively; and the constant $\kappa>0$ represents the thermal diffusivity; $f$ denote the external force.

The initial data is given by

$$
\rho(x, 0)=\rho_{0}(x), \rho u(x, 0)=\rho_{0} u_{0}(x), \theta(x, 0)=\theta_{0}(x), x \in \mathbb{R}^{2} .
$$

Equation (1.1) governs the motions of the incompressible nonisothermal viscous capillary fluids. Assumed capillarity coefficient $v=0$, the system (1.1) can simplify to other incompressible equations. Recently, the incompressible equations with density $\rho>0$ have led scholars to do much research, which gets important results in different academic fields. The study of the system (1.1) with $\mu>0$ and $\kappa>0$ is more concerned. Cannon and DiBenedetto proved the initial value problem of the Boussinesq equation for incompressible fluid affected by convective heat transfer (see [1]). Furthermore, they improved the regularity of the solution when the initial data are smooth. In addition, few scholars further studied the cases of "partial viscosity" (i.e. either the zero diffusivity case, $\mu>0$ and $\kappa=0$, or the zero viscosity case, $\mu=0$ and $\kappa>0$ ). Hou and Li [2] demonstrated the global well-posedness of the Cauchy problem of viscous Boussinesq equations. Chae [3] considered the Boussinesq system for incompressible fluid in $\mathbb{R}^{2}$ with either zero diffusion $(\kappa=0)$ or zero viscosity $(\mu=0)$. He proved global-in-time regularity in both cases. In addition, the singularity problem of Equation (1.1) is still an unsolved problem in mathematical fluid mechanics. Under the generalized Boussinesq equation approximation, by giving the viscosity and thermal conductivity related to temperature, Lorca [4] and Boldrini [5] showed the initial value problem of viscous incompressible systems. In some recent studies, the density-dependent viscous incompressible Boussinesq system caused wide attention. Qiu and Yao [6] get the local well-posedness for the density-dependent Boussinesq Equation (1.1) and consider the regularity problem of the smooth solutions for this equation in Besov spaces. The paper [7] considers the stability and zero dissipation limit of the boundary problem of the multidimensional Boussinesq system. But, when the initial conditions include a vacuum situation, there are little relevant researches. For Equation (1.1) we propose the relationship between velocity field, fluid temperature and pressure so as to solve the difficulties caused by vacuum.

If the thermal diffusivity $\kappa=0$, the system (1.1) is referred to as Korteweg model. Research on the compressible Navier-Stokes-Korteweg fluid model has been developed. For small initial data, [8] [9] provided the existence problems of the global strong solutions for Korteweg system in Besov space. And the global existence of weak solutions in the whole space $\mathbb{R}^{2}$ was obtained by DanchinDesjardins [10] and Haspot [11]. For large initial data, Bresch-Desjardins-Lin [12] analyzed the Korteweg-type compressible fluid model with density-dependent 
capillary coefficient, and obtained existence results. Recently, Germain-LeFloch [13] studied the existence, convergence and compactness of the compressible Navier-Stokes-Korteweg model. And both the vacuum and nonvacuum weak solutions were obtained. Moreover, Chen-He-Zhao [14] discussed the construction of smooth solutions to the Cauchy problem of the fluid models of Korteweg type, and the global solvability results are acquired if the $\mu, v$ and $\kappa$ satisfy certain conditions (see also [14] [15]). Assuming that the influence of temperature is not considered, the Equation (1.1) can be simplified into a general incompressible Korteweg model, then liu-wang-zheng [16] studied the strong solution of Cauchy problem in this model. Up to now, when the Korteweg term is introduced into Boussinesq equation, this kind of problem is still unknown. For this problem, we have to consider the difficulty of Korteweg term $v \rho \nabla \Delta \rho$. More importantly, the particularity of vacuum state should also be considered. These are also the core issues of this article.

Our purpose is to study the Cauchy problem for the strong solutions of Equations ((1.1), (1.2)). For convenience, we can set $f=0$. Since

$$
\rho \nabla \Delta \rho=\nabla(\rho \Delta \rho)-\nabla \rho \Delta \rho,
$$

classifying the term $-v \nabla(\rho \Delta \rho)$ as the pressure term, we can deform the equation as

$$
\left\{\begin{array}{l}
\rho_{t}+u \cdot \nabla \rho=0, \\
\rho u_{t}+\rho u \cdot \nabla u+\nabla p=\mu \Delta u-v \nabla \rho \Delta \rho, \\
\theta_{t}+u \cdot \nabla \theta-\kappa \Delta \theta=0, \\
\operatorname{div} u=0, \quad(x, t) \in \mathbb{R}^{2} \times \mathbb{R}^{+} .
\end{array}\right.
$$

Now, we explain the estimation of the complex term in this model. It is worth noting that when the initial data meets (1.7), the uniqueness and existence result of the strong solution of (1.1)-(1.2) Cauchy problem has been discussed in [17]. In order to extend the local situation to large-time, we need not only lower order estimate on strong solution of (1.1)-(1.2), but also a priori estimates with higher norm. In this article, the estimate of terms $\left\|\rho^{1 / 2} u\right\|_{L^{2}\left(\mathbb{R}^{2}\right)},\|\nabla u\|_{L^{2}\left(\mathbb{R}^{2}\right)}$ and $u \cdot \nabla \theta$ requires us to use some special ideas. We follow the ideas of Lü-Shi-Zhong [18] studying the incompressible N-S equation and Lü-Xu-Zhong [17] studying the compressible MHD equation. First, we attempt to estimate on the $L^{\infty}\left(0, T ; L^{2}\left(\mathbb{R}^{2}\right)\right)$-norm of $\nabla u$ and $\nabla \theta$. Using the key technique of [19] [20] [21], we multiplied $\dot{u} \Delta=u_{t}+u \cdot \nabla u$ and abandoned the normal $u_{t}$ (see [20]). More importantly, motivated by [22] [23], the term $\int p \partial_{j} u^{i} \partial_{i} u^{j} \mathrm{~d} x$ was controlled by the basic theories of Hardy and BMO in the second section, which have the term $\|p\|_{\text {Вмо }}\left\|\partial_{j} u^{i} \partial_{i} u^{j}\right\|_{\mathcal{H}^{1}}$ (see (3.13)). Next, we use the Stokes system (3.18) to get the $\left\|\nabla^{2} u\right\|_{L^{r}}$ and $\|\nabla p\|_{L^{r}}$ (see (3.19)), the key point is to use Gagliardo-Nirenberg inequality to estimate the value of $\|\nabla \rho \Delta \rho\|_{L^{2}}$. Multiplying (1.1) $)_{3}$ by $\Delta \theta$ can control the strong coupled term $u \cdot \nabla \theta$ after integration by parts (see (3.16)). And, considered [17] [24] [25] [26], we apply $\partial_{t}+u \cdot \nabla$ to $(1.1)_{2}^{j}$ and multiply 
the resultant equality by $\dot{u}^{j}$ to attain the $L^{\infty}\left(0, T ; L^{2}\left(\mathbb{R}^{2}\right)\right)$-norm of $t^{1 / 2} \rho^{1 / 2} \dot{u}$ and the $L^{2}\left(\mathbb{R}^{2} \times(0, T)\right)$-norm of $t^{1 / 2} \nabla \dot{u}$ (see (3.31)), then together with (3.44) to attain $\left\|\rho \bar{x}^{a}\right\|_{W^{2, q}}$. Based on the above treatment of the special term, one can complete the higher order estimates of the solution $(\rho, u, p, \theta)$. Finally, motivated by [17], our new observation of this paper is to obtain the $L^{2}$-norm of $\bar{x}^{1 / 2} \theta$ and $\bar{x}^{1 / 2} \nabla \theta$ (see (3.76)), which are critical to constraint the $L^{2}\left(\mathbb{R}^{2} \times(0, T)\right)$-norm of both $t^{1 / 2} \nabla u_{t}$ and $t^{1 / 2} \nabla \theta_{t}$ and the $L^{\infty}\left(0, T ; L^{2}\left(\mathbb{R}^{2}\right)\right)$ norm of $t^{1 / 2} \nabla^{2} \theta$, see Lemma 3.8.

Now we will explain the symbols and conventions applied in this article. For $R>0$, let

$$
B_{R} \triangleq\left\{x \in^{2}|| x \mid<R\right\}, \int \cdot \mathrm{d} x \triangleq \int_{2} \cdot \mathrm{d} x
$$

Meanwhile, for $1 \leq r \leq \infty$ and $k \geq 1$, the standard Lebesgue and Sobolve spaces have the following forms:

$$
L^{r}=L^{r}\left(\mathbb{R}^{2}\right), \quad W^{k, r}=W^{k, r}\left(\mathbb{R}^{2}\right), \quad H^{k}=W^{k, 2} .
$$

Next, we show the definition of strong solution to system (1.1) as follows:

Definition 1.1. Assumed the whole derivatives related to system for $(\rho, u, p, \theta)$ are regular distributions, Equation (1.1) also hold almost everywhere in $\mathbb{R}^{2} \times(0, T)$, then $(\rho, u, p, \theta)$ is considered a strong solution to (1.1).

Moreover, it can be assumed that the initial density $\rho_{0}$ satisfies

$$
\int_{\mathbb{R}^{2}} \rho_{0} \mathrm{~d} x=1,
$$

which implies that exists a positive constant $N_{0}$ such that

$$
\int_{B_{N_{0}}} \rho_{0} \mathrm{~d} x \geq \frac{1}{2} \int \rho_{0} \mathrm{~d} x=\frac{1}{2} .
$$

The main conclusions of this paper are given as follows:

Theorem 1.2. Besides (1.5) and (1.6), if the initial data $\left(\rho_{0}, u_{0}, \theta_{0}\right)$ hold that for any constant $a>1$ and $q>2$,

$$
\left\{\begin{array}{l}
\rho_{0} \geq 0, \rho_{0} \bar{x}^{a} \in L^{1} \cap H^{2} \cap W^{2, q}, \nabla u_{0} \in L^{2}, \sqrt{\rho_{0}} u_{0} \in L^{2}, \\
\theta_{0} \geq 0, \theta_{0} \bar{x}^{\frac{a}{2}} \in L^{2}, \nabla \theta_{0} \in L^{2}, \operatorname{div} u_{0}=0,
\end{array}\right.
$$

where

$$
\bar{x} \triangleq\left(e+|x|^{2}\right)^{\frac{1}{2}} \log ^{2}\left(e+|x|^{2}\right)
$$

and satisfy the compatibility condition

$$
-\mu \Delta u_{0}+\nabla p_{0}+\nu \nabla \rho_{0} \Delta \rho_{0}=\sqrt{\rho_{0}} g,
$$

for some $p_{0} \in H^{1}\left(B_{R}\right)$ and $g \in L^{2}\left(B_{R}\right)$, $\operatorname{div} u_{0}=0$. Then the problem (1.1)(1.2) has a unique global strong solution $(\rho, u, p, \theta)$ satisfying that for any $0<T<\infty$, 


$$
\left\{\begin{array}{l}
0 \leq \rho \in C\left([0, T] ; L^{1} \cap H^{2} \cap W^{2, q}\right), \\
\rho \bar{x}^{a} \in L^{\infty}\left(0, T ; L^{2} \cap H^{1} \cap W^{2, q}\right), \\
\sqrt{\rho} u, \nabla u, \bar{x}^{-1} u, \sqrt{t} \sqrt{\rho} u_{t}, \sqrt{t} \nabla p, \sqrt{t} \nabla^{2} u \in L^{\infty}\left(0, T ; L^{2}\right), \\
\theta, \theta \bar{x}^{a / 2}, \nabla \theta, \sqrt{t} \theta_{t}, \sqrt{t} \nabla^{2} \theta, \sqrt{t} \nabla \theta \bar{x}^{a / 2} \in L^{\infty}\left(0, T ; L^{2}\right), \\
\nabla u \in L^{2}\left(0, T ; H^{1}\right) \cap L^{(q+1) / q}\left(0, T ; W^{1, q}\right), \\
\nabla p \in L^{2}\left(0, T ; L^{2}\right) \cap L^{(q+1) / q}\left(0, T ; L^{q}\right), \\
\nabla \theta \in L^{2}\left(0, T ; H^{1}\right), \theta_{t}, \nabla \theta \bar{x}^{a / 2} \in L^{2}\left(0, T ; L^{2}\right), \\
\sqrt{t} \nabla u \in L^{2}\left(0, T ; W^{1, q}\right), \\
\sqrt{\rho} u_{t}, \sqrt{t} \nabla u_{t}, \sqrt{t} \nabla \theta_{t}, \sqrt{t} \bar{x}^{-1} u_{t} \in L^{2}\left(\mathbb{R}^{2} \times(0, T)\right),
\end{array}\right.
$$

and

$$
\inf _{0 \leq t \leq T} \int_{B_{N_{1}}} \rho(x, t) \mathrm{d} x \geq \frac{1}{4}
$$

for positive constant $N_{1}$ depending only $\left\|\rho_{0}\right\|_{L^{1}},\left\|\rho_{0}^{\frac{1}{2}} u_{0}\right\|_{L^{2}}, N_{0}$ and $T$. In addition, the $(\rho, u, p, \theta)$ has the following decay rates, that is for $t \geq 1$,

$$
\left\{\begin{array}{l}
\|\nabla u(\cdot, t)\|_{L^{2}}+\|\nabla \theta(\cdot, t)\|_{L^{2}} \leq C t^{-1 / 2}, \\
\left\|\nabla^{2} u(\cdot, t)\right\|_{L^{2}}+\|\nabla p(\cdot, t)\|_{L^{2}} \leq C t^{-1},
\end{array}\right.
$$

where $\mathrm{C}$ depends only on $\mu, \kappa,\left\|\rho_{0}\right\|_{L^{1} \cap L^{\infty}},\left\|\rho_{0}^{1 / 2} u_{0}\right\|_{L^{2}},\left\|\nabla u_{0}\right\|_{L^{2}}$, and $\left\|\theta_{0}\right\|_{H^{1}}$.

Remark 1.3. If there is no influence of fluid temperature, i.e., $\theta=0$, then (1.1) reduces to the fluid of Korteweg type, Theorem 1.2 extends the results of Liu and Wang [27] to the Cauchy problem of global solutions in two-dimensional space. When the initial data is large, there is no other compatibility conditions are considered for the global existence of the strong solutions.

The following sections of the article are introduced as follows: first, in Section 2, we give some basic facts and important inequalities, which can be applied in the calculations below. Next, in Section 3, we will give the priori estimates. In Section 4, we will attain the important result of this paper, Theorem 1.2, based on the previous.

\section{Preliminaries}

In this section, we recall the relevant results obtained by previous mathematicians and state our main results. Then, we begin with the unique and local strong solution. As follows:

Lemma 2.1. If that $\left(\rho_{0}, u_{0}, \theta_{0}\right)$ satisfies (1.7). Then there exists a small time $T_{0}>0$ and a unique strong solution $(\rho, u, p, \theta)$ to the problem (1.1)-(1.2) in $\mathbb{R}^{2} \times\left(0, T_{0}\right)$ that satisfies (1.10) and (1.11).

Lemma 2.2. (Gagliardo-Nirenberg inequality). For $m \in[2, \infty), \quad q \in(1, \infty)$, and $r \in(2, \infty)$, there exists some generic constant $C>0$ which may depend 
on $\mathrm{m}$, q, and r such that for $f \in H^{1}\left(\mathbb{R}^{2}\right)$ and $g \in L^{q}\left(\mathbb{R}^{2}\right) \cap D^{1, r}\left(\mathbb{R}^{2}\right)$, we have

$$
\begin{gathered}
\|f\|_{L^{m}\left(\mathbb{R}^{2}\right)}^{m} \leq C\|f\|_{L^{2}\left(\mathbb{R}^{2}\right)}^{2}\|\nabla f\|_{L^{2}\left(\mathbb{R}^{2}\right)}^{m-2}, \\
\|g\|_{C\left(\overline{\mathbb{R}^{2}}\right)} \leq C\|g\|_{L^{q}\left(\mathbb{R}^{2}\right)}^{q((2-))((2 r+q(r-2))}\|\nabla g\|_{L^{r}\left(\mathbb{R}^{2}\right)}^{2 r /(r r q(r-2))} .
\end{gathered}
$$

The next weighted $L^{n}$ bounds can be seen in ([28], Theorem 1.1) for elements in $\tilde{D}^{1,2}\left(\mathbb{R}^{2}\right) \triangleq\left\{v \in H_{\text {loc }}^{1}\left(\mathbb{R}^{2}\right) \mid \nabla v \in L^{2}\left(\mathbb{R}^{2}\right)\right\}$.

Lemma 2.3. For $h \in[2, \infty)$ and $\lambda \in(1+h / 2, \infty)$, there exists a positive constant $\mathrm{C}$ such that for all $v \in \tilde{D}^{1,2}\left(\mathbb{R}^{2}\right)$,

$$
\begin{aligned}
& \left(\int_{\mathbb{R}^{2}} \frac{|v|^{h}}{e+|x|^{2}}\left(\log \left(e+|x|^{2}\right)\right)^{-\lambda} \mathrm{d} x\right)^{1 / h} \\
& \leq C\|v\|_{L^{2}\left(B_{1}\right)}+C\|\nabla v\|_{L^{2}\left(\mathbb{R}^{2}\right)} .
\end{aligned}
$$

Between Lemma 2.3 and the Poincaré inequality, we can get the following key results on weighted bounds, this proof is mentioned in ([18], Lemma 2.4).

Lemma 2.4. Let $\bar{x}$ be as in (1.8). Assume that $\rho \in L^{1}\left(\mathbb{R}^{2}\right) \cap L^{\infty}\left(\mathbb{R}^{2}\right)$ is a non-negative function such that

$$
\|\rho\|_{L^{1}\left(B_{N_{0}}\right)} \geq M_{0}, \quad\|\rho\|_{L^{1}\left(\mathbb{R}^{2}\right) \cap L^{\infty}\left(\mathbb{R}^{2}\right)} \leq M_{1},
$$

for positive constants $M_{0}, M_{1}$, and $N_{0} \geq 1$ with $B_{N_{0}} \subset \mathbb{R}^{2}$. Then for $\alpha>0$, $\beta>0$, there is a positive constant $C$ depending only on $\alpha, \beta, M_{0}, M_{1}$, and $N_{0}$ such that every $v \in \tilde{D}^{1,2}\left(\mathbb{R}^{2}\right)$ satisfies

$$
\left\|v \bar{X}^{-\beta}\right\|_{L^{(2+\alpha) / \tilde{\beta}}\left(\mathbb{R}^{2}\right)} \leq C\left\|\rho^{1 / 2} v\right\|_{L^{2}\left(\mathbb{R}^{2}\right)}+C\|\nabla v\|_{L^{2}\left(\mathbb{R}^{2}\right)},
$$

with $\tilde{\beta}=\min \{1, \beta\}$.

Finally, set $\mathcal{H}^{1}\left(\mathbb{R}^{2}\right)$ and $\mathrm{BMO}\left(\mathbb{R}^{2}\right)$ denote the standard Hardy and BMO spaces (see [29], chapter IV). Then the next basic fact is very important for proving lemma 3.2 in the section 3.

Lemma 2.5 (i) There is a positive constant $C$ such that

$$
\|G \cdot M\|_{\mathcal{H}^{1}\left(\mathbb{R}^{2}\right)} \leq C\|G\|_{L^{2}\left(\mathbb{R}^{2}\right)}\|M\|_{L^{2}\left(\mathbb{R}^{2}\right)},
$$

for all $G \in L^{2}\left(\mathbb{R}^{2}\right)$ and $M \in L^{2}\left(\mathbb{R}^{2}\right)$ satisfying

$$
\operatorname{div} G=0, \quad \nabla^{\perp} \cdot M=0 \quad \text { in } D^{\prime}\left(\mathbb{R}^{2}\right) .
$$

(ii) There is a positive constant $\mathrm{C}$ such that

$$
\|f\|_{\mathrm{BMO}\left(\mathbb{R}^{2}\right)} \leq C\|\nabla f\|_{L^{2}\left(\mathbb{R}^{2}\right)},
$$

for all $f \in \tilde{D}^{1,2}\left(\mathbb{R}^{2}\right)$.

Proof. (i) For the specific proof steps, please see ([30], theorem II.1).

(ii) It follows from the Poincaré inequality that for any ball $B \subset\left(\mathbb{R}^{2}\right)$

$$
\frac{1}{|B|} \int_{B}\left|v(x)-\frac{1}{|B|} \int_{B} v(y) \mathrm{d} y\right| \mathrm{d} x \leq C\left(\int_{B}|\nabla v|^{2} \mathrm{~d} x\right)^{1 / 2},
$$




\section{A Priori Estimates of the Solution}

\section{Lower Order Estimates}

First, because of $\operatorname{div} u=0$, we have the following estimate related to the density on the $L^{\infty}\left(0, T ; L^{r}\right)$-norm.

Lemma 3.1. There exists a positive constant $C$ depending only on $\left\|\rho_{0}\right\|_{L^{1} \cap L^{\infty}}$ such that

$$
\sup _{t \in[0, T]}\|\rho\|_{L^{1} \cap L^{\infty}} \leq C .
$$

We give the time-independent estimates of $\nabla u$ and $\nabla \theta$ on the $L^{\infty}\left(0, T ; L^{2}\right)$ -norm.

Lemma 3.2. There exists a positive constant $C$ depending only on $\mu, \kappa$, $\left\|\rho_{0}\right\|_{L^{\infty}},\left\|\nabla u_{0}\right\|_{L^{2}},\left\|\sqrt{\rho_{0}} u_{0}\right\|_{L^{2}}$, and $\left\|\theta_{0}\right\|_{H^{1}}$ such that

$$
\begin{aligned}
& \sup _{t \in[0, T]}\left(\|\nabla u\|_{L^{2}}^{2}+\|\nabla \theta\|_{L^{2}}^{2}+\|\theta\|_{L^{2}}^{2}\right)+\int_{0}^{T}\left(\|\sqrt{\rho} \dot{u}\|_{L^{2}}^{2}+\|\Delta \theta\|_{L^{2}}^{2}\right) \mathrm{d} t \\
& \leq C \int_{0}^{T}\|\nabla \dot{u}\|_{L^{2}}^{2} \mathrm{~d} t+C \int_{0}^{T} \psi \mathrm{d} t,
\end{aligned}
$$

Here $\dot{u} \triangleq \partial_{t} u+u \cdot \nabla u$, and $\psi=\left\|\rho \bar{x}^{a}\right\|_{L^{1} \cap H^{2} \cap W^{2, q}}^{2}$, furthermore, one has

$$
\begin{aligned}
& \sup _{t \in[0, T]} t\left(\|\nabla u\|_{L^{2}}^{2}+\|\nabla \theta\|_{L^{2}}^{2}\right)+\int_{0}^{T} t\left(\left\|\rho^{\frac{1}{2}} \dot{u}\right\|_{L^{2}}^{2}+\|\Delta \theta\|_{L^{2}}^{2}\right) \mathrm{d} t \\
& \leq C \int_{0}^{T}\|\nabla \dot{u}\|_{L^{2}}^{2} \mathrm{~d} t+C \int_{0}^{T} \psi \mathrm{d} t .
\end{aligned}
$$

Proof. Applying standard energy estimate, taking the $x_{i}$-derivative $(i=1,2)$ of (1.4) gives

$$
\partial_{i} \rho_{t}+\partial_{i} u \cdot \nabla \rho+u \cdot \partial_{i}(\nabla \rho)=0 .
$$

Multiplying (1.1) by $2 \nabla \rho$ and integrating the resulting equality on $\mathbb{R}^{2}$, we get

$$
\frac{\mathrm{d}}{\mathrm{d} t}\|\nabla \rho\|_{L^{2}}^{2}+C\|\nabla u\|_{L^{2}}^{2}+C\|\nabla \rho\|_{L^{4}}^{4} \leq C .
$$

Adding (1.4) $\times 2 u$ to $(1.4) \times u^{2}$ and integrating the resulting equality on $\mathbb{R}^{2}$, we have

$$
\frac{\mathrm{d}}{\mathrm{d} t}\|\sqrt{\rho} u\|_{L^{2}}^{2}+2 \mu\|\nabla u\|_{L^{2}}^{2} \leq C\|\nabla u\|_{L^{2}}^{2}+C\|\nabla \rho\|_{L^{4}}^{4} .
$$

Multiplying (1.1) by $\theta$ and integrating the resulting equality on $\mathbb{R}^{2}$, we have

$$
\frac{\mathrm{d}}{\mathrm{d} t}\|\theta\|_{L^{2}}^{2}+\|\nabla \theta\|_{L^{2}}^{2} \leq C .
$$

Combining (3.5) with (3.6), (3.7), and then integrating on $[0, T]$ gives

$$
\sup _{t \in[0, T]}\left(\|\sqrt{\rho} u\|_{L^{2}}^{2}+\|\nabla \rho\|_{L^{2}}^{2}+\|\theta\|_{L^{2}}^{2}\right)+\int_{0}^{T}\left(\|\nabla u\|_{L^{2}}^{2}+\|\nabla \theta\|_{L^{2}}^{2}\right) \mathrm{d} t \leq C .
$$

Next, multiplying (1.1) by $\dot{u}$ and integrating the resulting equality on $\mathbb{R}^{2}$, 
we have

$$
\begin{aligned}
\int \rho|\dot{u}|^{2} \mathrm{~d} x & =\int \mu \Delta u \cdot \dot{u} \mathrm{~d} x-\int \nabla p \cdot \dot{u} \mathrm{~d} x-v \int \nabla \rho \Delta \rho \cdot \dot{u} \mathrm{~d} x \\
& \triangleq F_{1}+F_{2}+F_{3} .
\end{aligned}
$$

Then it follows from integration by parts and Gagliardo-Nirenberg inequality that

$$
\begin{aligned}
F_{1} & =\mu \int \Delta u \cdot\left(u_{t}+u \cdot \nabla u\right) \mathrm{d} x \\
& =-\frac{\mu}{2} \frac{\mathrm{d}}{\mathrm{d} t}\|\nabla u\|_{L^{2}}^{2}-\mu \int \partial_{i} u^{j} \partial_{i}\left(u^{k} \partial_{k} u^{j}\right) \mathrm{d} x \\
& \leq-\frac{\mu}{2} \frac{\mathrm{d}}{\mathrm{d} t}\|\nabla u\|_{L^{2}}^{2}+C\|\nabla u\|_{L^{3}}^{3} \\
& \leq-\frac{\mu}{2} \frac{\mathrm{d}}{\mathrm{d} t}\|\nabla u\|_{L^{2}}^{2}+C\|\nabla u\|_{L^{2}}^{2}\left\|\nabla^{2} u\right\|_{L^{2}} .
\end{aligned}
$$

Integrating by parts together with (1.1) gives

$$
F_{2}=-\int \nabla p\left(u_{t}+u \cdot \nabla u\right) \mathrm{d} x=\int p \partial_{j} u^{i} \partial_{i} u^{j} \mathrm{~d} x \leq C\|p\|_{\mathrm{BMO}}\left\|\partial_{j} u^{i} \partial_{i} u^{j}\right\|_{\mathcal{H}^{1}},
$$

where one has used the duality of $\mathcal{H}^{1}$ space and BMO one (see [28], Chapter IV]) in the last inequality. Since $\operatorname{div}\left(\partial_{j} u\right)=\partial_{j} \operatorname{div} u=0, \nabla^{\perp} \cdot\left(\nabla u^{j}\right)=0$, and (2.6) yields

$$
\left\|\partial_{j} u^{i} \partial_{i} u^{j}\right\|_{\mathcal{H}^{1}} \leq C\|\nabla u\|_{L^{2}}\|\nabla u\|_{L^{2}} \cdot
$$

Equation (3.11) combined with Equation (3.12) and Equation (2.8) gives

$$
\left|F_{2}\right|=\left|\int p \partial_{j} u^{i} \partial_{i} u^{j} \mathrm{~d} x\right| \leq C\|p\|_{\mathrm{BMO}}\|\nabla u\|_{L^{2}}^{2} \leq C\|\nabla p\|_{L^{2}}\|\nabla u\|_{L^{2}}^{2} .
$$

Integration by parts together with (1.4), (3.4), (3.8), and Gagliardo-Nirenberg inequality gives

$$
\begin{aligned}
F_{3} & =\frac{v}{2} \int(\nabla \rho)^{2} \nabla \dot{u} \mathrm{~d} x \leq C\|\nabla \rho\|_{L^{4}}^{4}+C\|\nabla \dot{u}\|_{L^{2}}^{2} \\
& \leq C\left\|\Delta\left(\bar{x}^{-a} \cdot \bar{x}^{a} \rho\right)\right\|_{L^{2}}^{2}+C\|\nabla \dot{u}\|_{L^{2}}^{2} \\
& \leq C\left\|\bar{x}^{a} \rho\right\|_{H^{2}}^{2}+C\|\nabla \dot{u}\|_{L^{2}}^{2} \leq C \psi+C\|\nabla \dot{u}\|_{L^{2}}^{2} .
\end{aligned}
$$

Next, substituting (3.10), (3.13) and (3.14) into (3.9) gives

$$
\frac{\mu}{2} \frac{\mathrm{d}}{\mathrm{d} t}\|\nabla u\|_{L^{2}}^{2}+\|\sqrt{\rho} \dot{u}\|_{L^{2}}^{2} \leq C\left(\left\|\nabla^{2} u\right\|_{L^{2}}+\|\nabla p\|_{L^{2}}\right)\|\nabla u\|_{L^{2}}^{2}+C\|\nabla \dot{u}\|_{L^{2}}^{2}+C \psi .
$$

Then, multiplying (1.1) by $\Delta \theta$ and integrating the resulting equality by parts over $\mathbb{R}^{2}$, it follows from Hölder's and Gagliardo-Nirenberg inequalities that

$$
\begin{aligned}
& \frac{\mathrm{d}}{\mathrm{d} t} \int|\nabla \theta|^{2} \mathrm{~d} x+2 \kappa \int|\Delta \theta|^{2} \mathrm{~d} x \\
& \leq C \int\left|\nabla u\left\|\left.\nabla \theta\right|^{2} \mathrm{~d} x \leq C\right\| \nabla u\left\|_{L^{3}}\right\| \nabla \theta\left\|_{L^{2}}^{\frac{4}{3}}\right\| \nabla \theta \|_{L^{2}}^{\frac{2}{3}}\right. \\
& \leq C\|\nabla u\|_{L^{2}}^{2}\left\|\nabla^{2} u\right\|_{L^{2}}+C\|\nabla \theta\|_{L^{2}}^{4}+C\|\Delta \theta\|_{L^{2}}^{2},
\end{aligned}
$$

which combined with (3.15) and (3.8) gives 


$$
\begin{aligned}
& \frac{\mathrm{d}}{\mathrm{d} t}\left(\frac{\mu}{2}\|\nabla u\|_{L^{2}}^{2}+\|\nabla \theta\|_{L^{2}}^{2}\right)+\|\sqrt{\rho} \dot{u}\|_{L^{2}}^{2}+2 \kappa\|\Delta \theta\|_{L^{2}}^{2} \\
& \leq C\|\nabla \theta\|_{L^{2}}^{4}+C\left(\left\|\nabla^{2} u\right\|_{L^{2}}+\|\nabla p\|_{L^{2}}\right)\|\nabla u\|_{L^{2}}^{2}+C\|\nabla \dot{u}\|_{L^{2}}^{2}+C \psi .
\end{aligned}
$$

Since $(\rho, u, p, \theta)$ satisfies the following Stokes system

$$
\begin{cases}-\mu \Delta u+\nabla p=-\rho \dot{u}-v \nabla \rho \Delta \rho, & x \in \mathbb{R}^{2}, \\ \operatorname{div} u=0, & x \in \mathbb{R}^{2}, \\ u(x) \rightarrow 0, & |x| \rightarrow \infty,\end{cases}
$$

Applying the standard $L^{r}$-estimate to (3.18) (see [31]) yields that for any $r>1$,

$$
\left\|\nabla^{2} u\right\|_{L^{r}}+\|\nabla p\|_{L^{r}} \leq C\|\rho \dot{u}\|_{L^{r}}+C\|\nabla \rho \Delta \rho\|_{L^{r}} \leq C\|\sqrt{\rho} \dot{u}\|_{L^{r}}+C\|\nabla \rho \Delta \rho\|_{L^{r}} .
$$

Moreover, since $\bar{x}^{-a}, \nabla \bar{x}^{-a}, \nabla^{2} \bar{x}^{-a}$ are all bounded in $\mathbb{R}^{2}$, an application of the Gagliardo-Nirenberg inequality results in

$$
\begin{aligned}
\|\nabla \rho \Delta \rho\|_{L^{2}} & \leq\|\nabla \rho\|_{L^{\infty}}\|\Delta \rho\|_{L^{2}} \leq C\|\nabla \rho\|_{W^{1, q}}^{\frac{q}{2(q-1)}}\|\nabla \rho\|_{L^{2}}^{\frac{q-2}{2(q-1)}}\|\Delta \rho\|_{L^{2}} \\
& =C\left\|\nabla\left(\bar{x}^{-a} \cdot \bar{x}^{a} \rho\right)\right\|_{W^{1, q}}^{\frac{q}{2(q-1)}}\left\|\nabla\left(\bar{x}^{-a} \cdot \bar{x}^{a} \rho\right)\right\|_{L^{2}}^{\frac{q-2}{2(q-1)}}\left\|\Delta\left(\bar{X}^{-a} \cdot \bar{x}^{a} \rho\right)\right\|_{L^{2}} \\
& \leq C\left\|\rho \bar{x}^{a}\right\|_{W^{2, q}}^{\frac{q}{2(q-1)}}\left\|\rho \bar{x}^{a}\right\|_{H^{1}}^{\frac{q-2}{2(q-1)}}\left\|\rho \bar{x}^{a}\right\|_{H^{2}} \leq C \psi,
\end{aligned}
$$

substituting (3.20) into (3.19), we get

$$
\left\|\nabla^{2} u\right\|_{L^{2}}+\|\nabla p\|_{L^{2}} \leq \varepsilon\|\sqrt{\rho} \dot{u}\|_{L^{2}}+C \psi .
$$

This combined with (3.17) and (3.19) gives

$$
\begin{aligned}
& \frac{\mathrm{d}}{\mathrm{d} t}\left(\frac{\mu}{2}\|\nabla u\|_{L^{2}}^{2}+\|\nabla \theta\|_{L^{2}}^{2}\right)+\|\sqrt{\rho} \dot{u}\|_{L^{2}}^{2}+2 \kappa\|\Delta \theta\|_{L^{2}}^{2} \\
& \leq C\|\nabla \theta\|_{L^{2}}^{4}+C\|\nabla u\|_{L^{2}}^{4}+\varepsilon\|\sqrt{\rho} \dot{u}\|_{L^{2}}^{2}+C\|\nabla \dot{u}\|_{L^{2}}^{2}+C \psi,
\end{aligned}
$$

where $\varepsilon$ is to be determined. Choosing $\varepsilon=\frac{1}{2}$, it follows from (3.22) that

$$
\begin{aligned}
& \frac{\mathrm{d}}{\mathrm{d} t}\left(\frac{\mu}{2}\|\nabla u\|_{L^{2}}^{2}+\|\nabla \theta\|_{L^{2}}^{2}\right)+\frac{1}{2}\|\sqrt{\rho} \dot{u}\|_{L^{2}}^{2}+2 \kappa\|\Delta \theta\|_{L^{2}}^{2} \\
& \leq C\left(\|\nabla u\|_{L^{2}}^{2}+\|\nabla \theta\|_{L^{2}}^{2}\right)\left(\|\nabla \theta\|_{L^{2}}^{2}+\|\nabla u\|_{L^{2}}^{2}+C\right)+C\|\nabla \dot{u}\|_{L^{2}}^{2}+C \psi,
\end{aligned}
$$

which together with (3.8), (3.22) and Gronwall's inequality gives (3.2). Then, multiplying (3.22) by $t$, we have

$$
\begin{aligned}
& \frac{\mathrm{d}}{\mathrm{d} t} t\left(\frac{\mu}{2}\|\nabla u\|_{L^{2}}^{2}+\|\nabla \theta\|_{L^{2}}^{2}\right)-\frac{\mu}{2}\|\nabla u\|_{L^{2}}^{2}-\|\nabla \theta\|_{L^{2}}^{2}+\frac{1}{2} t\|\sqrt{\rho} \dot{u}\|_{L^{2}}^{2}+2 \kappa t\|\Delta \theta\|_{L^{2}}^{2} \\
& \leq t\left(\|\nabla u\|_{L^{2}}^{2}+\|\nabla \theta\|_{L^{2}}^{2}\right)\left(\|\nabla \theta\|_{L^{2}}^{2}+\|\nabla u\|_{L^{2}}^{2}+C\right)+C\|\nabla \dot{u}\|_{L^{2}}^{2}+C \psi
\end{aligned}
$$

which combined (3.8) with Gronwall's inequality gives (3.3). Finally, the above completes the proof of Lemma 3.2.

Lemma 3.3. There exists a positive constant $\mathrm{C}$ depending only on $\mu$, 


$$
\begin{gathered}
\left\|\nabla u_{0}\right\|_{L^{2}},\left\|\rho_{0}\right\|_{L^{1} \cap L^{\infty}},\left\|\rho_{0}^{\frac{1}{2}} u_{0}\right\|_{L^{2}},\left\|\rho_{0} \bar{x}^{a}\right\|_{L^{1}}, \quad N_{0} \text {, and T, such that } \\
\sup _{t \in[0, T]}\left\|\rho \bar{x}^{a}\right\|_{L^{1}} \leq C(T) .
\end{gathered}
$$

Proof. First, for $N>1$, let $\varphi_{N} \in C_{0}^{\infty}\left(B_{N}\right)$ satisfy

$$
0 \leq \varphi_{N} \leq 1, \quad \varphi_{N}(x)=\left\{\begin{array}{ll}
1, & |x| \leq N / 2, \\
0, & |x| \geq N,
\end{array} \quad\left|\nabla \varphi_{N}\right| \leq C N^{-1} .\right.
$$

It follows from (1.1) that

$$
\begin{aligned}
\frac{\mathrm{d}}{\mathrm{d} t} \int \rho \varphi_{N} \mathrm{~d} x & =\int \rho u \cdot \nabla \varphi_{N} \mathrm{~d} x \\
& \geq-C N^{-1}\left(\int \rho \mathrm{d} x\right)^{1 / 2}\left(\int \rho|u|^{2} \mathrm{~d} x\right)^{1 / 2} \\
& \geq-\tilde{C} N^{-1}
\end{aligned}
$$

where in the last inequality one has used (3.1) and (3.8). Integrating (3.27) and choosing $N=N_{1} \triangleq 2 N_{0}+4 \tilde{C} T$, we obtain after using (1.6) that

$$
\begin{aligned}
\inf _{t \in[0, T]} \int_{B_{N_{1}}} \rho \mathrm{d} x & \geq \inf _{t \in[0, T]} \int \rho \varphi_{N_{1}} \mathrm{~d} x \geq \int \rho_{0} \varphi_{N_{1}} \mathrm{~d} x-\tilde{C} N_{1}^{-1} T \\
& \geq \int_{B_{N_{0}}} \rho_{0} \mathrm{~d} x-\frac{\tilde{C} T}{2 N_{0}+4 \tilde{C} T} \geq \frac{1}{4} .
\end{aligned}
$$

Hence, it follows from (3.28), (3.1), (2.2), (3.8) and (3.2) that for any $\eta \in(0,1]$ and any $s>2$,

$$
\left\|u \bar{x}^{-\eta}\right\|_{L^{s / \eta}} \leq C\left(\left\|\rho^{1 / 2} u\right\|_{L^{2}}+\|\nabla u\|_{L^{2}}\right)
$$

Multiplying (1.1) by $\bar{x}^{a}$ and integrating the resulting equality by parts over $\mathbb{R}^{2}$ yield that

$$
\begin{aligned}
\frac{\mathrm{d}}{\mathrm{d} t} \int \rho \bar{x}^{a} \mathrm{~d} x & \leq C \int \rho|u| \bar{x}^{a-1} \log ^{2}\left(e+|x|^{2}\right) \mathrm{d} x \\
& \leq C\left\|\rho \bar{x}^{a-1+\frac{8}{8+a}}\right\|_{\frac{8+a}{L^{7+a}}}\left\|u \bar{x}^{-\frac{4}{8+a}}\right\|_{L^{8+a}} \\
& \leq C \int \rho \bar{x}^{a} \mathrm{~d} x+C,
\end{aligned}
$$

this together with Gronwall's inequality can get (3.25), and the above completes the proof of Lemma 3.3.

Lemma 3.4. There exists a positive constant $C$ depending only on $\mu, \kappa$, $\left\|\rho_{0}\right\|_{L^{1} \cap L^{\infty}},\left\|\nabla u_{0}\right\|_{L^{2}}$ and $\left\|\sqrt{\rho_{0}} u_{0}\right\|_{L^{2}}$ such that for $i=1,2$,

$$
\sup _{t \in[0, T]} t^{i}\|\sqrt{\rho} \dot{u}\|_{L^{2}}^{2}+\int_{0}^{T} t^{i}\|\nabla \dot{u}\|_{L^{2}}^{2} \mathrm{~d} t \leq C \int_{0}^{T} \psi \mathrm{d} t
$$

and

$$
\sup _{t \in[0, T]} t^{i}\left(\left\|\nabla^{2} u\right\|_{L^{2}}^{2}+\|\nabla p\|_{L^{2}}^{2}\right) \leq C \int_{0}^{T} \psi \mathrm{d} t .
$$

Proof. Operating $\partial_{t}+u \cdot \nabla$ to $(1.1)^{j}$, one gets by some simple calculations that 


$$
\begin{aligned}
& \partial_{t}\left(\rho \dot{u}^{j}\right)+\operatorname{div}\left(\rho u \dot{u}^{j}\right)-\mu \Delta \dot{u}^{j} \\
& =-\mu \partial_{i}\left(\partial_{i} u \cdot \nabla u^{j}\right)-\mu \operatorname{div}\left(\partial_{i} u \partial_{i} u^{j}\right)-\partial_{j} \partial_{t} p-(u \cdot \nabla) \partial_{j} p \\
& -v \partial_{t}(\nabla \rho \Delta \rho)-v(u \cdot \nabla)(\nabla \rho \Delta \rho) .
\end{aligned}
$$

Next, multiplying (3.33) by $\dot{u}^{j}$, together with integration by parts and (1.1), we get

$$
\begin{aligned}
& \frac{1}{2} \frac{\mathrm{d}}{\mathrm{d} t} \int \rho|\dot{u}|^{2} \mathrm{~d} x+\mu \int|\nabla \dot{u}|^{2} \mathrm{~d} x \\
& =-\int \mu \partial_{i}\left(\partial_{i} u \cdot \nabla u^{j}\right) u^{j} \mathrm{~d} x-\int \mu \operatorname{div}\left(\partial_{i} u \partial_{i} \dot{u}^{j}\right) \dot{u}^{j} \mathrm{~d} x \\
& \quad-\int\left(\dot{u}^{j} \partial_{t} \partial_{j} p+\dot{u}^{j}(u \cdot \nabla) \partial_{j} p\right) \mathrm{d} x-v \int \dot{u}^{j}\left(\partial_{t}\left(\partial_{i} \rho \Delta \rho\right)+(u \cdot \nabla)\left(\partial_{i} \rho \Delta \rho\right)\right) \mathrm{d} x \\
& \triangleq \sum_{i=1}^{4} H_{i}
\end{aligned}
$$

Following the same argument as ([21], Lemma 3.3) we have the estimates of $\left(H_{i}(i=1,2,3)\right)$ as

$$
\sum_{i=1}^{3} H_{i} \leq \frac{\mathrm{d}}{\mathrm{d} t} \int p \partial_{j} u^{i} \partial_{i} u^{j} \mathrm{~d} x+C\left(\|p\|_{L^{4}}^{4}+\|\nabla u\|_{L^{4}}^{4}\right)+\frac{\varepsilon}{2}\|\nabla \dot{u}\|_{L^{2}}^{2} .
$$

which combined (1.3), (3.4) and the Gagliardo-Nirenberg inequality leads to

$$
\begin{aligned}
H_{4} & =-v \int \nabla \rho_{t} \Delta \rho \cdot \dot{u}^{j} \mathrm{~d} x-v \int \nabla \rho \Delta \rho_{t} \cdot \dot{u}^{j} \mathrm{~d} x-v \int(u \cdot \nabla)(\nabla \rho \Delta \rho) \cdot \dot{u}^{j} \mathrm{~d} x \\
& =v \int \nabla \rho \nabla \rho_{t} \nabla \dot{u} \mathrm{~d} x+v \int(u \cdot \nabla) \rho \Delta \rho \nabla \dot{u} \mathrm{~d} x \\
& \leq v \int|\nabla \rho|^{2}|\nabla u \| \nabla \dot{u}| \mathrm{d} x \\
& \leq C\left\|\Delta\left(\bar{x}^{-a} \cdot \bar{x}^{a} \rho\right)\right\|_{L^{2}}^{6}+C\|\nabla u\|_{L^{4}}^{4}+C\|\nabla \dot{u}\|_{L^{2}}^{2} \\
& \leq C\|\nabla u\|_{L^{4}}^{4}+\frac{\varepsilon}{2}\|\nabla \dot{u}\|_{L^{2}}^{2}+C \psi .
\end{aligned}
$$

Substituting (3.35) and (3.36) into (3.34) and together with (3.2) gives

$$
\frac{\mathrm{d}}{\mathrm{d} t} A(t)+\frac{\mu}{2}\|\nabla \dot{u}\|_{L^{2}}^{2} \leq C\left(\|p\|_{L^{4}}^{4}+\|\nabla u\|_{L^{4}}^{4}\right)+C \psi,
$$

where

$$
A(t) \triangleq \frac{1}{2} \int \rho|\dot{u}|^{2} \mathrm{~d} x-\int p \partial_{j} u^{i} \partial_{i} u^{j} \mathrm{~d} x,
$$

satisfies

$$
\frac{1}{4}\left\|\rho^{\frac{1}{2}} \dot{u}\right\|_{L^{2}}^{2}-\varepsilon\|\nabla u\|_{L^{2}}^{4}+C \psi \leq A(t) \leq \frac{1}{4}\left\|\rho^{\frac{1}{2}} \dot{u}\right\|_{L^{2}}^{2}+\varepsilon\|\nabla u\|_{L^{2}}^{4}+C \psi .
$$

Due to (3.13) and (3.21), for the right-hand side of (3.37), it follows from (3.13), (3.21), (3.1) and Sobolev's inequality that

$$
\begin{aligned}
\|p\|_{L^{4}}^{4}+\|\nabla u\|_{L^{4}}^{4} & \leq C\left(\|\nabla p\|_{L^{\frac{4}{3}}}^{4}+\left\|\nabla^{2} u\right\|_{L^{\frac{4}{3}}}^{4}\right) \leq C\|\rho \dot{u}\|_{L^{\frac{4}{3}}}^{4}+C \psi^{2} \\
& \leq C\|\rho\|_{L^{2}}^{2}\|\sqrt{\rho} \dot{u}\|_{L^{2}}^{4}+C \psi^{2} \leq C\|\sqrt{\rho} \dot{u}\|_{L^{2}}^{4}+C \psi^{2} .
\end{aligned}
$$

Substituting (3.38) and (3.39) into (3.37) 


$$
\begin{aligned}
& \frac{\mathrm{d}}{\mathrm{d} t} A(t)+\frac{\mu}{2}\|\nabla \dot{u}\|_{L^{2}}^{2} \\
& \leq C\|\sqrt{\rho} \dot{u}\|_{L^{2}}^{4}+C \psi^{2} \leq C\left(\|\sqrt{\rho} \dot{u}\|_{L^{2}}^{2}+C \psi\right)\left(A(t)+\|\nabla u\|_{L^{2}}^{4}\right) \\
& \leq C\left(\|\sqrt{\rho} \dot{u}\|_{L^{2}}^{2}+C \psi\right) A(t)+C\left(\|\sqrt{\rho} \dot{u}\|_{L^{2}}^{2}+\|\nabla u\|_{L^{2}}^{4}+C \psi\right) .
\end{aligned}
$$

Next, we estimate $\|\sqrt{\rho} \dot{u}\|_{L^{2}}^{2}$. First, the (3.25) combined (3.8) with (3.29) that for any $\eta>0$ and $\sigma>0$

$$
\begin{aligned}
\left\|\rho^{\eta} u\right\|_{L^{\sigma}} & \leq\left\|\rho^{\eta} \bar{x}^{\frac{3 a}{4 \sigma}}\right\|_{L^{\frac{4 \sigma}{3}}}\left\|u \bar{x}^{-\frac{3 a}{4 \sigma}}\right\|_{L^{4 \sigma}} \\
& \leq C\|\rho\|_{L^{\infty}}^{\eta-\frac{3}{4 \sigma}}\left\|\rho \bar{x}^{a}\right\|_{L^{1}}^{\frac{3}{4 \sigma}}\left\|_{\bar{x}^{-}-\frac{3 a}{4 \sigma}}\right\|_{L^{4 \sigma}} \\
& \leq C\left(\|\sqrt{\rho} u\|_{L^{2}}+\|\nabla u\|_{L^{2}}\right) .
\end{aligned}
$$

The (1.4) combined with (3.8), (3.41) and the Gagliardo-Nirenberg inequality, we derive

$$
\begin{aligned}
\int \rho|\dot{u}|^{2} \mathrm{~d} x \leq & C \int \rho\left|u_{t}\right|^{2} \mathrm{~d} x+C \int \rho[(u \cdot \nabla u)]^{2} \mathrm{~d} x \\
\leq & C \int \rho|u|^{2}|\nabla u|^{2} \mathrm{~d} x+C \int \rho^{-1}|\mu \Delta u-\nabla p-\kappa \nabla \rho \Delta \rho|^{2} \mathrm{~d} x+\|\rho u\|_{L^{4}}^{2}\|\nabla u\|_{L^{4}}^{2} \\
\leq & C \int \rho|u|^{2}|\nabla u|^{2} \mathrm{~d} x+C \int \rho^{-1}|\mu \Delta u-\nabla p-\kappa \nabla \rho \Delta \rho|^{2} \mathrm{~d} x \\
& +C\left(\|\sqrt{\rho} u\|_{L^{2}}^{2}+\|\nabla u\|_{L^{2}}^{2}\right)\|\nabla u\|_{L^{2}}\left(\|\nabla u\|_{L^{2}}+\left\|\nabla^{2} u\right\|_{L^{2}}\right) \\
\leq & C \int \rho|u|^{2}|\nabla u|^{2} \mathrm{~d} x+C \int \rho^{-1}|\mu \Delta u-\nabla p-\kappa \nabla \rho \Delta \rho|^{2} \mathrm{~d} x+\varepsilon\|\nabla u\|_{L^{2}}^{2}+C \psi,
\end{aligned}
$$

which together with (3.41) and the compatibility condition (1.9) yields

$$
\begin{aligned}
& \int \rho|\dot{u}|^{2}(x, 0) \mathrm{d} x \\
& \leq \sup _{t \rightarrow 0^{+}} \int C\left(\rho^{-1}|\mu \Delta u-\nabla p-\kappa \nabla \rho \Delta \rho|^{2}+\rho|u|^{2}|\nabla u|^{2}\right) \mathrm{d} x+\varepsilon\|\nabla u\|_{L^{2}}^{2}+C \psi \\
& \leq C(T)\|g\|_{L^{2}}^{2}+C\left\|\rho_{0} u_{0}\right\|_{L^{4}}^{2}\left\|\nabla u_{0}\right\|_{L^{4}}^{2}+\varepsilon\left\|\nabla u_{0}\right\|_{L^{2}}^{2}+C \psi \\
& \leq C(T)\|g\|_{L^{2}}^{2}+C\left(\left\|\sqrt{\rho_{0}} u_{0}\right\|_{L^{2}}^{2}+\left\|\nabla u_{0}\right\|_{L^{2}}^{2}\right)\left\|\nabla u_{0}\right\|_{L^{2}}\left\|\nabla u_{0}\right\|_{H^{1}} \\
& \leq C(T) .
\end{aligned}
$$

Finally, Multiplying (3.40) by $t^{i}(i=1,2)$ and using (3.43), it deduces from Gronwalls inequality and (3.8) to lead to (3.31). The (3.32) is a direct result of (3.31) and (3.21). The proof of Lemma 3.4 is finished.

Lemma 3.5. There exists a positive constant $\mathrm{C}$ depending on $\mathrm{T}$ such that

$$
\begin{aligned}
& \sup _{t \in[0, T]}\|\rho\|_{H^{1} \cap W^{1, q}}+\int_{0}^{T}\left(\left\|\nabla^{2} u\right\|_{L^{2}}^{2}+\left\|\nabla^{2} u\right\|_{L^{q}}^{q+1}+t\left\|\nabla^{2} u\right\|_{L^{2} \cap L^{q}}^{2}\right) \mathrm{d} t \\
& +\int_{0}^{T}\left(\|\nabla p\|_{L^{2}}^{2}+\|\nabla p\|_{L^{q}}^{\frac{q+1}{q}}+t\|\nabla p\|_{L^{2} \cap L^{q}}^{2}\right) \mathrm{d} t \leq C \int_{0}^{T} \psi^{2} \mathrm{~d} t .
\end{aligned}
$$

Proof. First, it follows from the mass Equation (1.1) that $\nabla \rho$ satisfies for any 
$r \geq 2$,

$$
\frac{\mathrm{d}}{\mathrm{d} t}\|\nabla \rho\|_{L^{r}} \leq C(r)\|\nabla u\|_{L^{\infty}}\|\nabla \rho\|_{L^{r}} .
$$

Next, employing Gagliardo-Nirenberg inequality, using (3.2) and (3.19), we have $q>2$,

$$
\|\nabla u\|_{L^{\infty}} \leq C\|\nabla u\|_{L^{2}}^{\frac{q-2}{2(q-1)}}\left\|\nabla^{2} u\right\|_{L^{q}}^{\frac{q}{2(q-1)}} \leq C\left(\|\rho \dot{u}\|_{L^{q}}^{\frac{q}{2(q-1)}}+\psi\right) .
$$

It follows from (3.28), (3.1), (2.2) and (3.25) that for any $s>2$,

$$
\begin{aligned}
\|\rho v\|_{L^{s}} & \leq C\left\|\rho \bar{X}^{\frac{3 a}{4 s}}\right\|\left\|_{L^{s}}\right\| v \bar{X}^{-\frac{3 a}{4 s}} \|_{L^{4 s}} \\
& \leq C\|\rho\|_{L^{\infty}}^{\frac{4 s}{4 s}}\left\|\rho \bar{X}^{a}\right\|_{L^{1}}^{\frac{3}{4 s}}\left(\left\|\rho^{1 / 2} v\right\|_{L^{2}}+\|\nabla v\|_{L^{2}}\right) \\
& \leq C\left(\left\|\rho^{1 / 2} v\right\|_{L^{2}}+\|\nabla v\|_{L^{2}}\right),
\end{aligned}
$$

which together with the Gagliardo-Nirenberg inequality shows that

$$
\begin{aligned}
\|\rho \dot{u}\|_{L^{q}} & \leq C\|\rho \dot{u}\|_{L^{2}}^{\frac{2(q-1)}{q^{2}-2}}\|\rho \dot{u}\|_{L^{q^{q^{2}}}}^{\frac{q(q-2)}{q^{2}-2}} \\
& \leq C\|\rho \dot{u}\|_{L^{2}}^{\frac{2(q-1)}{q^{2}-2}}\left(\|\sqrt{\rho} \dot{u}\|_{L^{2}}+\|\nabla \dot{u}\|_{L^{2}}\right)^{\frac{q(q-2)}{q^{2}-2}} \\
& \leq C\left(\|\sqrt{\rho} \dot{u}\|_{L^{2}}+\|\sqrt{\rho} \dot{u}\| \frac{2(q-1)}{q_{L^{2}-2}^{2}}\|\nabla \dot{u}\|_{L^{2}}^{\frac{q(q-2)}{q^{2}-2}}\right),
\end{aligned}
$$

which is deformed and calculated appropriately leads to

$$
\begin{aligned}
\int_{0}^{T}\|\rho \dot{u}\|_{L^{q}}^{\frac{q+1}{q}} \mathrm{~d} t & \leq C \int_{0}^{T}\|\sqrt{\rho} \dot{u}\|_{L^{2}}^{\frac{q+1}{q}} \mathrm{~d} t+\sup _{t \in[0, T]}\left(t\|\sqrt{\rho} \dot{u}\|_{L^{2}}^{2}\right)^{\frac{q^{2}-1}{2 q\left(q^{2}-2\right)}} \\
& \cdot \int_{0}^{T} t^{-\frac{q^{3}+q^{2}-2 q-2}{2 q\left(q^{2}-2\right)}}\left(t\|\nabla \dot{u}\|_{L^{2}}^{2}\right)^{\frac{q(q-2)(q+1)}{2 q\left(q^{2}-2\right)}} \mathrm{d} t \\
\leq & C \int_{0}^{T}\|\sqrt{\rho} \dot{u}\|_{L^{2}}^{2} \mathrm{~d} t+C \int_{0}^{T} t^{-\frac{q^{3}+q^{2}-2 q-2}{q^{3}+q^{2}-2 q}} \mathrm{~d} t+C \int_{0}^{T} t\|\nabla \dot{u}\|_{L^{2}}^{2} \mathrm{~d} t \\
\leq & \int_{0}^{T} \psi^{2} \mathrm{~d} t, \\
\int_{0}^{T} t & \|\rho \dot{u}\|_{L^{q}}^{2} \mathrm{~d} t \leq \int_{0}^{T}\left(t\|\nabla \dot{u}\|_{L^{2}}^{2}+\psi\right) \mathrm{d} t \leq C \int_{0}^{T} \psi^{2} \mathrm{~d} t .
\end{aligned}
$$

Then, the (3.49) along with (3.46) in particular implies

$$
\int_{0}^{T}\|\nabla u\|_{L^{\infty}} \mathrm{d} t \leq C \int_{0}^{T} \psi^{2} \mathrm{~d} t .
$$

Next, applying Gronwall's inequality to (3.45) gives

$$
\sup _{t \in[0, T]}\|\nabla \rho\|_{L^{2} \cap L^{q}} \leq C \int_{0}^{T} \psi^{2} \mathrm{~d} t .
$$

Setting $r=2$ in (3.19) and integrating the resulting equality over $[0, T]$, we obtain after using (3.1), (3.2) and (3.3) that 


$$
\int_{0}^{T}\left\|\nabla^{2} u\right\|_{L^{2}}^{2} \mathrm{~d} t+\int_{0}^{T}\|\nabla p\|_{L^{2}}^{2} \mathrm{~d} t \leq C \int_{0}^{T} \psi^{2} \mathrm{~d} t
$$

Similarly, setting $r=q$ in (3.19) and integrating the resulting equality over $[0, T]$, we deduce from using (3.49), (3.1), (3.2) and (3.3) that

$$
\int_{0}^{T}\left\|\nabla^{2} u\right\|_{L^{q}}^{\frac{q+1}{q}} \mathrm{~d} t+\int_{0}^{T}\|\nabla p\|_{L^{q}}^{\frac{q+1}{q}} \mathrm{~d} t \leq C \int_{0}^{T} \psi^{2} \mathrm{~d} t .
$$

Multiplying (3.19) by $t$ and integrating the resulting equality over $[0, T]$, it can obtain after using (3.50), (3.1), (3.2) and (3.3) that

$$
\int_{0}^{T} t\left\|\nabla^{2} u\right\|_{L^{2} \cap L^{q}}^{2} \mathrm{~d} t+\int_{0}^{T} t\|\nabla p\|_{L^{2} \cap L^{q}}^{2} \mathrm{~d} t \leq C \int_{0}^{T} \psi^{2} \mathrm{~d} t .
$$

Furthermore, it is easy to deduce from (3.53), (3.54) and (3.55) that

$$
\begin{aligned}
& \int_{0}^{T}\left(\left\|\nabla^{2} u\right\|_{L^{2}}^{2}+\left\|\nabla^{2} u\right\|_{L^{q}}^{\frac{q+1}{q}}+t\left\|\nabla^{2} u\right\|_{L^{2} \cap L^{q}}^{2}\right) \mathrm{d} t \\
& +\int_{0}^{T}\left(\|\nabla p\|_{L^{2}}^{2}+\|\nabla p\|_{L^{q}}^{\frac{q+1}{q}}+t\|\nabla p\|_{L^{2} \cap L^{q}}^{2}\right) \mathrm{d} t \leq C \int_{0}^{T} \psi^{2} \mathrm{~d} t,
\end{aligned}
$$

this together with (3.1) and (3.52) yields (3.44), which completes the proof of Lemma 3.5.

Lemma 3.6. There exists a positive constant $C$ depending on $T$ such that for $q>2$,

$$
\sup _{t \in[0, T]}\left\|\rho \bar{x}^{a}\right\|_{L^{1} \cap H^{2} \cap W^{2, q}} \leq C(T) .
$$

Proof. First, setting $\rho=\rho \bar{x}^{a}$ in (1.1) that satisfies

$$
\partial_{t}\left(\rho \bar{x}^{a}\right)+u \cdot \nabla\left(\rho \bar{x}^{a}\right)-a \rho \bar{x}^{a} u \cdot \nabla \log \bar{x}=0 .
$$

Taking the $x_{i}$-derivative on both side of the (3.58) gives

$$
\begin{aligned}
0= & \partial_{t} \partial_{i}\left(\rho \bar{x}^{a}\right)+u \cdot \nabla \partial_{i}\left(\rho \bar{x}^{a}\right)+\partial_{i} u \cdot \nabla\left(\rho \bar{x}^{a}\right)-a \partial_{i}\left(\rho \bar{x}^{a}\right) u \cdot \nabla \log \bar{x} \\
& -a \rho \bar{x}^{a} \partial_{i} u \cdot \nabla \log \bar{x}-a \rho \bar{x}^{a} u \cdot \partial_{i} \nabla \log \bar{x} .
\end{aligned}
$$

For any $r \in[2, q]$, multiplying (3.59) by $\left|\nabla\left(\rho \bar{x}^{a}\right)\right|^{r-2} \partial_{i}\left(\rho \bar{x}^{a}\right)$ and integrating the resulting equality by parts over $\mathbb{R}^{2}$, we obtain that

$$
\begin{aligned}
& \frac{\mathrm{d}}{\mathrm{d} t}\left\|\nabla\left(\rho \bar{x}^{a}\right)\right\|_{L^{r}} \\
& \leq C\left(1+\|\nabla u\|_{L^{\infty}}+\|u \cdot \nabla \log \bar{x}\|_{L^{\infty}}\right)\left\|\nabla\left(\rho \bar{x}^{a}\right)\right\|_{L^{r}} \\
& +C\left\|\rho \bar{x}^{a}\right\|_{L^{\infty}}\left(\|\nabla u\| \nabla \log \bar{x}\left\|_{L^{r}}+\right\| u\left\|\nabla^{2} \log \bar{x}\right\|_{L^{r}}\right) \\
& \leq C\left(1+\|\nabla u\|_{W^{1, q}}\right)\left\|\nabla\left(\rho \bar{x}^{a}\right)\right\|_{L^{r}}+C\left\|\rho \bar{x}^{a}\right\|_{L^{\infty}}\left(\|\nabla u\|_{L^{r}}+\left\|u \bar{x}^{-\frac{3}{5}}\right\|_{L^{4 r}}\left\|\bar{X}^{-\frac{4}{3}}\right\|_{L^{\frac{4 r}{3}}}\right) \\
& \leq C\left(1+\|\nabla u\|_{W^{1, q}}\right)\left(1+\left\|\nabla\left(\rho \bar{x}^{a}\right)\right\|_{L^{r}}+\left\|\nabla\left(\rho \bar{x}^{a}\right)\right\|_{L^{q}}\right),
\end{aligned}
$$

where in the last and the second inequalities, we has applied (3.29) and (3.25), respectively. Choosing $r=q$ (3.60), and applying Gronwall's inequality together with (3.44) indicates that 


$$
\sup _{t \in[0, T]}\left\|\nabla\left(\rho \bar{x}^{a}\right)\right\|_{L^{q}} \leq C .
$$

Setting $r=2$ in (3.60), we deduce from (3.44) and (3.61) that

$$
\sup _{t \in[0, T]}\left\|\nabla\left(\rho \bar{x}^{a}\right)\right\|_{L^{2}} \leq C .
$$

Next, taking the $x_{i}$-derivative again on both side of the (3.59) gives

$$
\begin{aligned}
0= & \partial_{i} \partial_{i}\left(\rho \bar{x}^{a}\right)_{t}+2 \partial_{i} u \cdot \partial_{i} \nabla\left(\rho \bar{x}^{a}\right)+u \cdot \partial_{i} \partial_{i} \nabla\left(\rho \bar{x}^{a}\right)+\partial_{i} \partial_{i} u \cdot \nabla\left(\rho \bar{x}^{a}\right) \\
& -a \partial_{i} \partial_{i}\left(\rho \bar{x}^{a}\right) u \cdot \nabla \ln \bar{x}-2 a \partial_{i}\left(\rho \bar{x}^{a}\right) \partial_{i} u \cdot \nabla \ln \bar{x}-2 a \partial_{i}\left(\rho \bar{x}^{a}\right) u \cdot \partial_{i} \nabla \ln \bar{x} \\
& -a \rho \bar{x}^{a} \partial_{i} \partial_{i} u \cdot \nabla \ln \bar{x}-2 a \rho \bar{x}^{a} \partial_{i} u \cdot \partial_{i} \nabla \ln \bar{x}-a \rho \bar{x}^{a} u \cdot \partial_{i} \partial_{i} \nabla \ln \bar{x} .
\end{aligned}
$$

Similarly, for any $p \in[2, q]$, multiplying (3.63) by $\left|\nabla^{2}\left(\rho \bar{x}^{a}\right)\right|^{p-2} \nabla^{2}\left(\rho \bar{x}^{a}\right)$ and integrating the resulting equality by parts over $\mathbb{R}^{2}$, we can find that

$$
\begin{aligned}
& \frac{\mathrm{d}}{\mathrm{d} t}\left\|\nabla^{2}\left(\rho \bar{x}^{a}\right)\right\|_{L^{p}} \\
& \leq C\left(1+\|\nabla u\|_{L^{\infty}}+\|u \cdot \nabla \ln \bar{x}\|_{L^{\infty}}\right)\left\|\nabla^{2}\left(\rho \bar{x}^{a}\right)\right\|_{L^{p}} \\
& +C\left\|\rho \bar{x}^{a}\right\|_{L^{\infty}}\left(\left\|\nabla^{2} u\right\|_{L^{p}}+\|\nabla u\| \nabla \ln \bar{x}\left\|_{L^{p}}+\right\| u\left\|\nabla^{2} \ln \bar{x}\right\|_{L^{p}}\right) \\
& +C\left\|\rho \bar{x}^{a}\right\|_{L^{\infty}}\left(\|\nabla u\| \nabla \ln \bar{x}\left\|_{L^{p}}+\right\| \nabla^{2} u\|\nabla \ln \bar{x}\|_{L^{p}}+\left\|u \cdot \nabla^{3} \ln \bar{x}\right\|_{L^{p}}\right) \\
& \leq C\left(1+\|\nabla u\|_{L^{2}}+\left\|\nabla^{2} u\right\|_{L^{q}}+\left\|u \bar{x}^{-\frac{1}{4}}\right\| \|_{L^{\infty}}\right)\left\|\nabla^{2}\left(\rho \bar{x}^{a}\right)\right\|_{L^{p}} \\
& \quad+C\left(\|\nabla u\|_{L^{p}}+\left\|\nabla^{2} u\right\|_{L^{p}}+\left\|u \bar{x}^{-\frac{3}{5}}\right\|\left\|_{L^{\infty}}\right\| \bar{x}^{-\frac{4}{3}}\|\|_{L^{p}}\right) \nabla^{2}\left(\rho \bar{x}^{a}\right) \|_{L^{2} \cap L^{p}} \\
& \quad+C\left(\|\nabla u\|_{L^{p}}+\left\|\nabla^{2} u\right\|_{L^{p}}+\left\|u \bar{x}^{-\frac{1}{4}}\right\|\left\|_{L^{\infty}}\right\| \frac{3}{2}\|\|_{L^{p}}\right)\left\|\nabla\left(\rho \bar{x}^{a}\right)\right\|_{L^{2} \cap L^{p}} \\
& \leq C\left(1+\left\|\nabla^{2} u\right\|_{L^{2} \cap L^{p}}\right)\left(\left\|\nabla^{2}\left(\rho \bar{x}^{a}\right)\right\|_{L^{p}}+\left\|\nabla^{2}\left(\rho \bar{x}^{a}\right)\right\|_{L^{2} \cap L^{p}}+\left\|\nabla\left(\rho \bar{x}^{a}\right)\right\|_{L^{2} \cap L^{p}}\right) \\
& \leq C(1+\psi)\left(\left\|\nabla^{2}\left(\rho \bar{x}^{a}\right)\right\|_{L^{p}}+\left\|\nabla^{2}\left(\rho \bar{x}^{a}\right)\right\|_{L^{2} \cap L^{p}}+\left\|\nabla\left(\rho \bar{x}^{a}\right)\right\|_{L^{2} \cap L^{p}}\right) .
\end{aligned}
$$

Using (3.64) for Gronwall's inequality, and according to (3.25), (3.44) and (3.61), we gain the desired estimate (3.57). It completes the proof of the Lemma 3.6 .

Lemma 3.7. There exists a positive constant $C$ such that

$$
\begin{gathered}
\sup _{t \in[0, T]}\left\|\theta \bar{x}^{\frac{a}{2}}\right\|_{L^{2}}^{2}+\int_{0}^{T}\left\|\nabla \theta \bar{x}^{\frac{a}{2}}\right\|_{L^{2}}^{2} \mathrm{~d} t \leq C(T), \\
\sup _{t \in[0, T]}\left(t\left\|\nabla \theta \bar{x}^{\frac{a}{2}}\right\|_{L^{2}}^{2}\right)+\int_{0}^{T} t\left\|\Delta \theta \bar{x}^{\frac{a}{2}}\right\|_{L^{2}}^{2} \mathrm{~d} t \leq C(T) .
\end{gathered}
$$

Proof. First, multiplying (1.1) by $\theta \bar{x}^{a}$ and integrating the resulting equality by parts over $\mathbb{R}^{2}$, we have 


$$
\begin{aligned}
\frac{1}{2} \frac{\mathrm{d}}{\mathrm{d} t}\left\|\theta \bar{x}^{\frac{a}{2}}\right\|_{L^{2}}^{2}+\kappa\left\|\nabla \theta \bar{x}^{\frac{a}{2}}\right\|_{L^{2}}^{2} & =\frac{\kappa}{2} \int|\theta|^{2} \Delta \bar{x}^{a} \mathrm{~d} x+\frac{1}{2} \int|\theta|^{2} u \cdot \nabla \bar{x}^{a} \mathrm{~d} x \\
& \triangleq \hat{M}_{1}+\hat{M}_{2},
\end{aligned}
$$

where

$$
\begin{aligned}
\hat{M}_{1} & =\frac{\kappa}{2} \int|\theta|^{2} \Delta \bar{x}^{a} \mathrm{~d} x \\
& \leq C \int|\theta|^{2} \bar{x}^{a} \bar{x}^{-2} \log ^{4}\left(e+|x|^{2}\right) \mathrm{d} x \\
& \leq C\left\|\theta \bar{x}^{\frac{a}{2}}\right\|_{L^{2}}^{2}, \\
\hat{M}_{2} & =\frac{1}{2} \int|\theta|^{2} u \cdot \nabla \bar{x}^{a} \mathrm{~d} x \\
& \leq C\left\|\theta \bar{x}^{\frac{a}{2}}\right\|_{L^{4}}\left\|\theta \bar{x}^{\frac{a}{2}}\right\|_{L^{2}}\left\|u \bar{x}^{-\frac{3}{4}}\right\|_{L^{4}} \\
& \leq C\left\|\theta \bar{x}^{\frac{a}{2}}\right\|_{L^{2}}^{2}+\frac{\kappa}{2}\left\|\nabla \theta \bar{x}^{\frac{a}{2}}\right\|_{L^{2}}^{2} .
\end{aligned}
$$

Substituting (3.68), (3.69) into (3.67), we get

$$
\frac{1}{2} \frac{\mathrm{d}}{\mathrm{d} t}\left\|\theta \bar{x}^{\frac{a}{2}}\right\|_{L^{2}}^{2}+\frac{\kappa}{2}\left\|\nabla \theta \bar{x}^{\frac{a}{2}}\right\|_{L^{2}}^{2} \leq C\left\|\theta \bar{x}^{\frac{a}{2}}\right\|_{L^{2}}^{2} .
$$

Using (3.70) for Gronwall's inequality, we obtain (3.65).

Next, we estimate the (3.66). Multiplying (1.1) by $\Delta \theta \bar{x}^{a}$ and integrating the resulting equality by parts on $\mathbb{R}^{2}$, we find

$$
\begin{aligned}
& \frac{1}{2} \frac{\mathrm{d}}{\mathrm{d} t}\left\|\nabla \theta \bar{x}^{\frac{a}{2}}\right\|_{L^{2}}^{2}+\kappa\left\|\Delta \theta \bar{x}^{\frac{a}{2}}\right\|_{L^{2}}^{2} \\
& \leq C \int\left|\nabla u \left\|\left.\nabla \theta\right|^{2} \bar{x}^{a} \mathrm{~d} x+C \int|u||\nabla \theta|^{2}\left|\nabla \bar{x}^{a}\right| \mathrm{d} x+C \int|\nabla \theta \| \Delta \theta|\left|\nabla \bar{x}^{a}\right| \mathrm{d} x\right.\right. \\
& \triangleq \sum_{i=1}^{3} \tilde{M}_{i},
\end{aligned}
$$

where

$$
\begin{aligned}
\tilde{M}_{1} & =C \int|\nabla u \| \nabla \theta|^{2} \bar{x}^{a} \mathrm{~d} x \\
& \leq C\|\nabla u\|_{L^{\infty}}\left\|\nabla \theta \bar{x}^{\frac{a}{2}}\right\|_{L^{2}}^{2} \\
& \leq C\|\nabla u\|_{L^{2}}^{\frac{q-2}{2(q-1)}}\left\|\nabla^{2} u\right\|_{L^{q}}^{\frac{q-2}{2(q-1)}}\left\|\nabla \theta \bar{x}^{\frac{a}{2}}\right\|_{L^{2}}^{2} \\
& \leq C\left(1+\left\|\nabla^{2} u\right\|_{L^{q}}^{\frac{q+1}{q}}\right)\left\|\nabla \theta \bar{x}^{\frac{a}{2}}\right\|_{L^{2}}^{2},
\end{aligned}
$$




$$
\begin{aligned}
\tilde{M}_{2} & =C \int|u||\nabla \theta|^{2}\left|\nabla \bar{x}^{a}\right| \mathrm{d} x \\
& \leq C\left\||\nabla \theta|^{2-\frac{2}{3 a}} \bar{x}^{a-\frac{1}{3}}\right\|_{L^{6 a-2}}\left\|u \bar{x}^{-\frac{1}{3}}\right\|_{L^{6 a}}\left\|\nabla \theta \mid \frac{2}{3 a}\right\|_{L^{6 a}} \\
& \leq C\left\|\nabla \theta \bar{x}^{\frac{a}{2}}\right\|_{L^{2}}^{\frac{6 a-2}{3 a}}\|\nabla \theta\|_{L^{4}}^{\frac{2}{3 a}} \\
& \leq C\left\|\nabla \theta \bar{x}^{\frac{a}{2}}\right\|_{L^{2}}^{2}+C\|\nabla \theta\|_{L^{4}}^{2} \\
& \leq C\left\|\nabla \theta \bar{x}^{\frac{a}{2}}\right\|_{L^{2}}^{2}+\frac{\kappa}{4}\left\|\Delta \theta \bar{x}^{\frac{a}{2}}\right\|_{L^{2}}^{2}, \\
\tilde{M}_{3}= & C \int\left|\nabla \theta\|\Delta \theta\| \nabla \bar{x}^{a}\right| \mathrm{d} x \leq \frac{\kappa}{4}\left\|\Delta \theta \bar{x}^{\frac{a}{2}}\right\|_{L^{2}}^{2}+C\left\|\nabla \theta \bar{x}^{\frac{a}{2}}\right\|_{L^{2}}^{2} .
\end{aligned}
$$

Submitting $\tilde{M}_{1}, \quad \tilde{M}_{2}, \tilde{M}_{3}$ into (3.71), one has

$$
\frac{1}{2} \frac{\mathrm{d}}{\mathrm{d} t}\left\|\nabla \theta \frac{\bar{x}}{\frac{a}{2}}\right\|_{L^{2}}^{2}+\frac{\kappa}{2}\left\|\Delta \theta \bar{x}^{\frac{a}{2}}\right\|_{L^{2}}^{2} \leq C\left(1+\left\|\nabla^{2} u\right\|_{L^{q}}^{\frac{q+1}{q}}\right)\left\|\nabla \theta \bar{x}^{\frac{a}{2}}\right\|_{L^{2}}^{2} .
$$

Multiplying (3.75) by $t$, and together with (3.65) and (3.44), then employing Gronwall's inequlity, one obtains the (3.66). This completes the Lemma 3.7.

Lemma 3.8. There exists a positive constant $C$ such that

$$
\sup _{t \in[0, T]} t\left(\left\|\rho^{\frac{1}{2}} u_{t}\right\|_{L^{2}}^{2}+\left\|\theta_{t}\right\|_{L^{2}}^{2}+\left\|\nabla^{2} \theta\right\|_{L^{2}}^{2}\right)+\int_{0}^{T}\left(t\left\|\nabla u_{t}\right\|_{L^{2}}^{2}+t\left\|\nabla \theta_{t}\right\|_{L^{2}}^{2}\right) \mathrm{d} t \leq C(T) .
$$

Proof. First, it is easy to deduce from (3.47), (3.29) that for any $\eta \in(0,1]$ and any $s>2$,

$$
\left\|\rho^{\eta} u\right\|_{L^{s / \eta}}+\left\|u \bar{x}^{-\eta}\right\|_{L^{s \eta}} \leq C
$$

Next, we prove

$$
\sup _{t \in[0, T]}\left(\|\nabla u\|_{L^{2}}^{2}+\|\nabla \theta\|_{L^{2}}^{2}\right)+\int_{0}^{T}\left(\left\|\rho^{\frac{1}{2}} u_{t}\right\|_{L^{2}}^{2}+\left\|\theta_{t}\right\|_{L^{2}}^{2}+\|\Delta \theta\|_{L^{2}}^{2}\right) \mathrm{d} t \leq C .
$$

With (3.2) at hand, we need only to show

$$
\int_{0}^{T}\left(\left\|\rho^{\frac{1}{2}} u_{t}\right\|_{L^{2}}^{2}+\left\|\theta_{t}\right\|_{L^{2}}^{2}\right) \mathrm{d} t \leq C .
$$

First, it is easy to show that

$$
\begin{aligned}
\left\|\rho^{\frac{1}{2}} u_{t}\right\|_{L^{2}}^{2} & \leq\left\|\rho^{\frac{1}{2}} \dot{u}\right\|_{L^{2}}^{2}+\left\|\rho^{\frac{1}{2}} \mid u\right\| \nabla u \|_{L^{2}}^{2} \\
& \leq\left\|\rho^{\frac{1}{2}} \dot{u}\right\|_{L^{2}}^{2}+C\left\|\rho^{\frac{1}{2}} u\right\|_{L^{6}}^{2}\|\nabla u\|_{L^{3}}^{2} \\
& \leq\left\|\rho^{\frac{1}{2}} \dot{u}\right\|_{L^{2}}^{2}+C\|\nabla u\|_{L^{2}}^{2}+C\left\|\nabla^{2} u\right\|_{L^{2}}^{2} .
\end{aligned}
$$


Then, due to (2.1) and (3.77), combining (2.1), (3.2) with (1.1)

$$
\left\|\theta_{t}\right\|_{L^{2}}^{2} \leq C\|\Delta \theta\|_{L^{2}}^{2}+\|u\| \nabla \theta\left\|_{L^{2}}^{2} \leq C\right\| \Delta \theta\left\|_{L^{2}}^{2}+\right\| \nabla \theta \bar{x}^{\frac{a}{2}} \|_{L^{2}}^{2},
$$

where in the last inequality one has used the follow facts

$$
\begin{aligned}
\|u\| \nabla \theta \|_{L^{2}}^{2} & \leq C\left\|u \bar{x}^{-\frac{a}{4}}\right\|_{L^{8}}^{4}\|\nabla \theta\|_{L^{4}}^{2}+C\left\|\nabla \theta \bar{x}^{\frac{a}{2}}\right\|_{L^{2}}^{2} \\
& \leq \frac{1}{2}\left\|\nabla^{2} \theta\right\|_{L^{2}}^{2}+C\left\|\nabla \theta \bar{x}^{\frac{a}{2}}\right\|_{L^{2}}^{2} .
\end{aligned}
$$

On the basis of (3.77) and (2.1), (3.79) can be derived by the combination of (3.80), (3.81), (3.44) and (3.65).

Next, differentiating $(1.1)_{2}$ with respect to $t$ shows

$$
\begin{aligned}
& \rho u_{t t}+\rho u \cdot \nabla u_{t}-\mu \Delta u_{t}+\nabla p_{t} \\
& =-\rho_{t}\left(u_{t}+u \cdot \nabla u\right)-\rho u_{t} \cdot \nabla u+v \Delta \rho \nabla(\nabla \rho \cdot u)+v \Delta(\nabla \rho \cdot u) \nabla \rho .
\end{aligned}
$$

Multiplying (3.83) by $u_{t}$ and integrating the resulting equality by parts on $\mathbb{R}^{2}$, it follows (1.1) that

$$
\begin{aligned}
& \frac{1}{2} \frac{\mathrm{d}}{\mathrm{d} t} \int \rho\left|u_{t}\right|^{2} \mathrm{~d} x+\mu \int\left|\nabla u_{t}\right|^{2} \mathrm{~d} x \\
& \leq C \int \rho|u|\left|u_{t}\right|\left(\nabla u_{t}+|\nabla u|^{2}+|u|\left|\nabla^{2} u\right|\right) \mathrm{d} x+C \int \rho|u|^{2}|\nabla u|\left|\nabla u_{t}\right| \mathrm{d} x \\
& \quad+C \int \rho\left|u_{t}\right|^{2}|\nabla u| \mathrm{d} x+2 v \int\left|\nabla^{2} \rho\right|^{2}|u|\left|u_{t}\right| \mathrm{d} x+v \int|\nabla \rho||\nabla u||\Delta \rho|\left|u_{t}\right| \mathrm{d} x \\
& \quad+v \int|\nabla \rho|\left|\nabla^{2} \rho\right||\nabla u|\left|u_{t}\right| \mathrm{d} x+v \int\left|\nabla^{2} \rho\right||\nabla \rho \| u|\left|\nabla u_{t}\right| \mathrm{d} x+v \int|\nabla \rho|^{2}|\nabla u|\left|\nabla u_{t}\right| \mathrm{d} x \\
& \triangleq \sum_{i=1}^{8} I_{i},
\end{aligned}
$$

where

$$
\begin{aligned}
& I_{1} \leq C\|\sqrt{\rho} u\|_{L^{6}}\left\|\sqrt{\rho} u_{t}\right\|_{L^{2}}^{\frac{1}{2}}\left\|\sqrt{\rho} u_{t}\right\|_{L^{6}}^{\frac{1}{2}}\left(\left\|\nabla u_{t}\right\|_{L^{2}}+\|\nabla u\|_{L^{4}}^{2}\right) \\
&+C\left\|\rho^{\frac{1}{4}} u\right\|_{L^{12}}^{2}\left\|\sqrt{\rho} u_{t}\right\|_{L^{2}}^{\frac{1}{2}}\left\|\sqrt{\rho} u_{t}\right\|_{L^{6}}^{\frac{1}{2}}\left\|\nabla^{2} u\right\|_{L^{2}} \\
& \leq C\left\|\sqrt{\rho} u_{t}\right\|_{L^{2}}^{\frac{1}{2}}\left(\left\|\sqrt{\rho} u_{t}\right\|_{L^{2}}+\left\|\nabla u_{t}\right\|_{L^{2}}\right)^{\frac{1}{2}}\left(\left\|\nabla u_{t}\right\|_{L^{2}}+\left\|\nabla^{2} u\right\|_{L^{2}}\right) \\
& \leq \varepsilon\left\|\nabla u_{t}\right\|_{L^{2}}^{2}+C\left(1+\left\|\sqrt{\rho} u_{t}\right\|_{L^{2}}^{2}+\left\|\nabla^{2} u\right\|_{L^{2}}^{2}\right), \\
& I_{2}+I_{3} \leq C\|\sqrt{\rho} u\|_{L^{8}}^{2}\|\nabla u\|_{L^{4}}\left\|\nabla u_{t}\right\|_{L^{2}}+\|\nabla u\|_{L^{2}}\left\|\sqrt{\rho} u_{t}\right\|_{L^{6}}^{\frac{3}{2}}\left\|\sqrt{\rho} u_{t}\right\|_{L^{2}}^{\frac{1}{2}} \\
& \leq \varepsilon\left\|\nabla u_{t}\right\|_{L^{2}}^{2}+C\left(1+\left\|\sqrt{\rho} u_{t}\right\|_{L^{2}}^{2}+\left\|\nabla^{2} u\right\|_{L^{2}}^{2}\right), \\
& I_{4}=2 v \int\left|\bar{x}^{a} \nabla^{2} \rho\right|^{2}\left|\bar{x}^{-a} u \| \bar{x}^{-a} u_{t}\right| d x \\
& \leq\left. 2 v\left\|\bar{x}^{a} \nabla^{2} \rho\right\|_{L^{q}}^{2}\left\|\bar{x}^{-a} u\right\|_{L^{2}}^{\frac{2 q}{q-2}}\left\|\bar{x}^{-a} u_{t}\right\|\right|_{L^{2-2}}
\end{aligned}
$$




$$
\begin{aligned}
& \leq C\left\|\rho \bar{x}^{a}\right\|_{W^{2, q}}^{2}\left(\|\sqrt{\rho} u\|_{L^{2}}+\|\nabla u\|_{L^{2}}\right)\left(\left\|\sqrt{\rho} u_{t}\right\|_{L^{2}}+\left\|\nabla u_{t}\right\|_{L^{2}}\right) \\
& \leq \varepsilon\left\|\nabla u_{t}\right\|_{L^{2}}^{2}+C\left\|\sqrt{\rho} u_{t}\right\|_{L^{2}}^{2} \text {, } \\
& I_{5}+I_{6} \leq 2 v \int|\nabla \rho|\left|\bar{x}^{a} \nabla^{2} \rho\right||\nabla u|\left|\bar{x}^{-a} u_{t}\right| \mathrm{d} x \\
& \leq 2 v\|\nabla \rho\|_{L^{2}}\|\nabla u\|_{L^{q^{q-2}}}\left\|\frac{4 q}{\bar{X}^{a}} \nabla^{2} \rho\right\|_{L^{q}}\left\|\bar{X}^{-a} u_{t}\right\| \frac{4 q}{L^{q-2}} \\
& \leq C\left\|\rho \bar{x}^{a}\right\|_{W^{2, q}}\left(\left\|\nabla^{2} u\right\|_{L^{2}}^{\frac{q+2}{2 q}}\|\nabla u\|_{L^{2}}^{\frac{q-2}{2 q}}+\|\nabla u\|_{L^{2}}\right)\left(\left\|\sqrt{\rho} u_{t}\right\|_{L^{2}}+\left\|\nabla u_{t}\right\|_{L^{2}}\right) \\
& \leq C\left(1+\left\|\nabla^{2} u\right\|_{L^{2}}^{\frac{q+2}{2 q}}\right)\left(\left\|\sqrt{\rho} u_{t}\right\|_{L^{2}}+\left\|\nabla u_{t}\right\|_{L^{2}}\right) \\
& \leq \varepsilon\left\|\nabla u_{t}\right\|_{L^{2}}^{2}+C\left(1+\left\|\sqrt{\rho} u_{t}\right\|_{L^{2}}^{2}+\left\|\nabla^{2} u\right\|_{L^{2}}^{2}\right), \\
& I_{7} \leq v\left\|\bar{X}^{a} \nabla^{2} \rho\right\|_{L^{q}}\left\|\bar{X}^{-a} u\right\|_{L^{q-2}}^{\frac{4 q}{q-2}}\|\nabla \rho\|_{L^{q-2}}^{\frac{4 q}{q-2}}\left\|\nabla u_{t}\right\|_{L^{2}} \\
& \leq C\left\|\rho \bar{X}^{a}\right\|_{W^{2, q}}\|\nabla \rho\|_{L^{2}}^{\frac{q-2}{2 q}}\|\nabla \rho\|_{H^{1}}^{\frac{q+2}{2 q}}\left\|\nabla u_{t}\right\|_{L^{2}} \\
& \leq \varepsilon\left\|\nabla u_{t}\right\|_{L^{2}}^{2}+C, \\
& I_{8} \leq v\|\nabla \rho\|_{L^{8}}^{2}\|\nabla u\|_{L^{4}}\left\|\nabla u_{t}\right\|_{L^{2}} \\
& \leq C\left(\|\nabla u\|_{L^{2}}^{\frac{1}{2}}\left\|\nabla^{2} u\right\|_{L^{2}}^{\frac{1}{2}}+\|\nabla u\|_{L^{2}}\right) \cdot\left(\|\nabla \rho\|_{L^{2}}^{\frac{1}{2}}\left\|\nabla^{2} \rho\right\|_{L^{2}}^{\frac{3}{2}}+\|\nabla \rho\|_{L^{2}}^{2}\right)\left\|\nabla u_{t}\right\|_{L^{2}} \\
& \leq C\left(\|\nabla u\|_{L^{2}}^{\frac{1}{2}}\left\|\nabla^{2} u\right\|_{L^{2}}^{\frac{1}{2}}+\|\nabla u\|_{L^{2}}\right) \cdot\left(\left\|\bar{X}^{a} \rho\right\|_{H^{1}}^{\frac{1}{2}}\left\|\bar{X}^{a} \rho\right\|_{H^{2}}^{\frac{3}{2}}+\left\|\bar{X}^{a} \rho\right\|_{H^{1}}^{2}\right)\left\|\nabla u_{t}\right\|_{L^{2}} \\
& \leq \varepsilon\left\|\nabla u_{t}\right\|_{L^{2}}^{2}+C\left(1+\left\|\nabla^{2} u\right\|_{L^{2}}\right),
\end{aligned}
$$

Submitting the above $I_{i}$ into (3.84) gives

$$
\frac{\mathrm{d}}{\mathrm{d} t}\left\|\rho^{\frac{1}{2}} u_{t}\right\|_{L^{2}}^{2}+C\left\|\nabla u_{t}\right\|_{L^{2}}^{2} \leq C\left\|\rho^{\frac{1}{2}} u_{t}\right\|_{L^{2}}^{2}+C\left(\left\|\nabla^{2} u\right\|_{L^{2}}^{2}+1\right) .
$$

Then, we multiply (3.91) by $t$, and together with Gronwall's inequality and (3.44) lead to

$$
\sup _{t \in[0, T]} t\left\|\rho^{\frac{1}{2}} u_{t}\right\|_{L^{2}}^{2}+\int_{0}^{T} t\left\|\nabla u_{t}\right\|_{L^{2}}^{2} \mathrm{~d} t \leq C .
$$

Next, differentiating (1.1) with respect to $t$ shows

$$
\theta_{t t}+u_{t} \cdot \nabla \theta+u \cdot \nabla \theta_{t}-\kappa \Delta \theta_{t}=0 .
$$

Now, multiplying (3.93) by $\theta_{t}$ and integrating the resulting equality by parts on $\mathbb{R}^{2}$, we find

$$
\begin{aligned}
& \frac{1}{2} \frac{\mathrm{d}}{\mathrm{d} t} \int\left|\theta_{t}\right|^{2} \mathrm{~d} x+\kappa \int\left|\nabla \theta_{t}\right|^{2} \mathrm{~d} x=\int u_{t} \cdot \nabla \theta_{t} \cdot \theta \mathrm{d} x \\
& \leq C\left\|\theta \bar{x}^{\frac{a}{2}}\right\|_{\frac{8 a}{4 a-1}}\left\|u_{t} \bar{x}^{-\frac{2 a-1}{4}}\right\|_{L^{8 a}}\left\|\nabla \theta_{t}\right\|_{L^{2}} \\
& \leq \frac{\mu}{2}\left(\left\|\nabla u_{t}\right\|_{L^{2}}^{2}+\left\|\rho^{\frac{1}{2}} u_{t}^{2}\right\|_{L^{2}}^{2}+\frac{\kappa}{2}\left\|\nabla \theta_{t}\right\|_{L^{2}}^{2} .\right.
\end{aligned}
$$


Next, multiplying (3.94) by $t$ and integrating the resulting equality by parts on $[0, T]$, it follows from $(3.79)$ that

$$
\sup _{t \in[0, T]} t\left\|\theta_{t}\right\|_{L^{2}}^{2}+\frac{\kappa}{2} \int_{0}^{T} t\left\|\nabla \theta_{t}\right\|_{L^{2}}^{2} \mathrm{~d} t \leq \frac{\mu}{2} \int_{0}^{T} t\left\|\nabla u_{t}\right\|_{L^{2}}^{2}+C .
$$

Finally, it follows from (1.1), and (3.82) that

$$
\begin{aligned}
\left\|\nabla^{2} \theta\right\|_{L^{2}}^{2} & \leq C\left\|\theta_{t}\right\|_{L^{2}}^{2}+C\|u\| \nabla \theta \|_{L^{2}}^{2} \\
& \leq C\left\|\theta_{t}\right\|_{L^{2}}^{2}+\frac{1}{2}\left\|\nabla^{2} \theta\right\|_{L^{2}}^{2}+C\left\|\nabla \theta \bar{x}^{\frac{a}{2}}\right\|_{L^{2}}^{2},
\end{aligned}
$$

which combined with (3.66), (3.92) and (3.95) indicates (3.76) and the proof of Lemma 3.8 is finished.

\section{Proof of Theorem 1.2}

In this Section, by the prior estimation in the previous chapter of Lemmas 3.1-3.8, we can complete the proof of Theorem 1.2.

Proof. On the basis of Lemmas 3.1-3.8, through previous result of local existence, there has a $T_{*}>0$ such that the Equation (1.1) and (1.2) have unique and local strong solution $(\rho, u, p, \theta)$ on $\mathbb{R}^{2} \times\left(0, T_{*}\right]$. Next, we will extend the local problem to all time.

Let

$$
T^{*}=\sup \left\{T \mid(\rho, u, p, \theta) \text { is a strong solution on } \mathbb{R}^{2} \times(0, T]\right\} \text {. }
$$

For any $0<\tau<T<T^{*}$ with $T$ finite, one deduces from (3.76) that for any $q \geq 2$,

$$
\nabla u, \nabla \theta, \theta \in C\left([\tau, T] ; L^{2} \cap L^{q}\right)
$$

where one has used the standard embedding

$$
L^{\infty}\left(\tau, T ; H^{1}\right) \cap H^{1}\left(\tau, T ; L^{2}\right) \hookrightarrow C\left([\tau, T] ; L^{q}\right) \text {, for any } q \in[2, \infty) .
$$

Moreover, it follows from (3.30), (3.56), and ([27], Lemma 2.3) that

$$
\rho \in C\left([0, T] ; L^{1} \cap H^{1} \cap W^{1, q}\right) .
$$

We claim that

$$
T^{*}=\infty \text {. }
$$

Otherwise, if $T^{*}<\infty$, it follows from (4.2), (4.3), (3.2), (3.8), (3.56), and (3.57) that

$$
(\rho, u, \theta)\left(x, T^{*}\right)=\lim _{t \rightarrow T^{*}}(\rho, u, \theta)(x, t)
$$

which satisfies the initial condition (1.6) at $t=T$. Thus, taking $(\rho, u, \theta)\left(x, T^{*}\right)$ as the initial data, since the existence and uniqueness of local strong solutions implies that there exists some $T^{* *}>T^{*}$, such that Theorem 1.2 satisfy $T=T^{* *}$. This contradicts the supposition of $T^{*}$ in (4.1), so the (4.4) holds. Therefore, the existence and uniqueness of local strong solutions and Lemmas 3.1-3.8 show 
that $(\rho, u, p, \theta)$ is in fact the unique strong solution on $\mathbb{R}^{2} \times[0, T]$ for any $0<T<T^{*}=\infty$. The above can prove Theorem 1.2.

\section{Conclusions}

For the general incompressible Navier-Stokes flow equation, there is no external force action, we can under the low estimate to a prior estimate of velocity and pressure. In this article, we study the two-dimensional incompressible Boussinesq the equations of Korteweg type model, and fluid temperature contains not only depends on the density of viscous coefficient, and influenced by external forces.

On the one hand, we should overcome the trouble of unbounded region when making the estimation, and carefully consider the special terms $\|\sqrt{\rho} u\|_{L^{2}}$ and $\|\nabla u\|_{L^{2}}$. At the same time, we should also consider the difficulties caused by the strong coupling between the velocity and temperature of the fluid. For example, $u \cdot \nabla \theta$, for such difficult terms, we should carry out ingenious structural analysis and strict calculation and derivation.

On the other hand, the Korteweg fluid model contains high order derivative terms of density, and the system we consider is in the case of large initial values, which makes it difficult to prove the global existence of strong solutions. In order to prove the global existence of the strong solution, we introduce the derivative of the random body and the auxiliary energy estimation of the fundamental inequality.

\section{Acknowledgments}

Thanks to those who contributed to this article but are not listed in the author list. Thanks to my tutor and classmates for their guidance and help on the model in this paper.

\section{Conflicts of Interest}

The author declares no conflicts of interest regarding the publication of this paper.

\section{References}

[1] Cannon, J. and DiBenedetto, E. (1980) The Initial Value Problem for the Boussinesq Equations with Data in $L^{p}$. In: Rautmann, R., Ed., Approximation Methods for Navier-Stokes Problems. Lecture Notes in Mathematics, Springer, Berlin, Heidelberg. https://doi.org/10.1007/BFb0086903

[2] Hou, T.Y. and Li, C. (2005) Global Well-Posedness of the Viscous Boussinesq Equations. Discrete \& Continuous Dynamical Systems, 12, 1-12. https://doi.org/10.3934/dcds.2005.12.1

[3] Chae, D. (2006) Global Regularity for the 2D Boussinesq Equations with Partial Viscosity Terms. Advances in Mathematics, 203, 497-513. https://doi.org/10.1016/j.aim.2005.05.001

[4] Lorca, S.A. and Boldrini, J.L. (1996) The Initial Value Problem for a Generalized 
Boussinesq Model: Regularity and Global Existence of Strong Solutions. Matemática Contemporânea, 11, 71-94.

[5] Lorca, S.A. and Boldrini, J.L. (1999) The Initial Value Problem for a Generalized Boussinesq Model. Nonlinear Analysis, 36, 457-480.

https://doi.org/10.1016/S0362-546X(97)00635-4

[6] Qiu, H. and Yao, Z. (2017) Well-Posedness for Density-Dependent Boussinesq Equations without Dissipation Terms in Besov Spaces. Computers \& Mathematics with Applications, 73, 1920-1931. https://doi.org/10.1016/j.camwa.2017.02.041

[7] Wang, J. and Xie, F. (2015) Zero Dissipation Limit and Stability of Boundary Layers for the Heat Conductive Boussinesq Equations in a Bounded Domain. Proceedings of the Royal Society of Edinburgh: Section A Mathematics, 145, 611-637. https://doi.org/10.1017/S0308210513000875

[8] Haspot, B. (2009) Existence of Strong Solutions for Nonisothermal Korteweg System. Annales Mathématiques Blaise Pascal, 16, 431-481.

https://doi.org/10.5802/ambp. 274

[9] Haspot, B. (2016) Existence of Global Strong Solution for Korteweg System with Large Infinite Energy Initial Data. Journal of Mathematical Analysis and Applications, 438, 395-443. https://doi.org/10.1016/j.jmaa.2016.01.047

[10] Danchin, R. and Desjardins, B. (2001) Existence of Solutions for Compressible Fluid Models of Korteweg Type. Annales de PInstitut Henri Poincaré C, Analyse non linéaire, 18, 97-133. https://doi.org/10.1016/S0294-1449(00)00056-1

[11] Haspot, B. (2011) Existence of Global Weak Solution for Compressible Fluid Models of Korteweg Type. Journal of Mathematical Fluid Mechanics, 13, 223-249. https://doi.org/10.1007/s00021-009-0013-2

[12] Bresch, D., Desjardins, B. and Lin, C.K. (2003) On Some Compressible Fluid Models: Korteweg, Lubrication and Shallow Water Systems. Communications in Partial Differential Equations, 28, 843-868. https://doi.org/10.1081/PDE-120020499

[13] Germain, P. and LeFloch, P.G. (2016) Finite Energy Method for Compressible Fluids: The Navie-Stokes-Korteweg Model. Communications on Pure and Applied Mathematics, 69, 3-61. https://doi.org/10.1002/cpa.21622

[14] Chen, Z., He, L. and Zhao, H. (2017) Global Smooth Solutions to the Nonisothermal Compressible Fluid Models of Korteweg Type with Large Initial Data. Zeitschrift für angewandte Mathematik und Physik, 68, Article No. 79. https://doi.org/10.1007/s00033-017-0822-8

[15] Chen, Z., Chai, X.J., Dong, B.Q. and Zhao, H.J. (2015) Global Classical Solutions to the One-Dimensional Compressible Fluid Models of Korteweg Type with Large Initial Data. Journal of Differential Equations, 259, 4376-4411.

https://doi.org/10.1016/j.jde.2015.05.023

[16] Liu, Y., Wang, W. and Zheng, S.N. (2018) Strong Solutions to the Cauchy Problem of Two-Dimensional Incompressible Fluid Models of Korteweg Type. Journal of Mathematical Analysis and Applications, 465, 1075-1093. https://doi.org/10.1016/j.jmaa.2018.05.054

[17] Lü, B.Q., Xu, Z.H. and Zhong, X. (2017) Global Existence and Large Time Asymptotic Behavior of Strong Solutions to the Cauchy Promblem of 2D Density-Dependent Magnetohydrodynamic Equations with Vacuum. Journal de Mathématiques Pures et Appliquées, 108, 41-62. https://doi.org/10.1016/j.matpur.2016.10.009

[18] Lü, B.Q., Shi, X.D. and Zhong, X. (2018) Global Existence and Large Time Asymptotic Behavior of Strong Solutions to the Cauchy Promblem of 2D Density-Dependent 
Navier-Stokes Equations with Vacuum. Nonlinearity, 31, 2617-2632. https://doi.org/10.1088/1361-6544/aab31f

[19] Li, J. and Xin, Z.P. (2019) Global Well-Posedness and Large Time Asymptotic Behavior of Classical Solutions to the Compressible Navier-Stokes Equations with Vacuum. Annals of PDE, 5, Article No. 37. https://doi.org/10.1007/s40818-019-0064-5

[20] Huang, X.D. and Wang, Y. (2014) Global Strong Solution with Vacuum to the Two-Dimensional Density-Dependent Navier-Stokes System. SIAM Journal on Mathematical Analysis, 46, 1771-1788. https://doi.org/10.1137/120894865

[21] Hoff, D. (1995) Global Solutions of the Navier-Stokes Equations for Multidimensional Compressible Flow with Discontinuous Initial Data. Journal of Differential Equations, 120, 215-254. https://doi.org/10.1006/jdeq.1995.1111

[22] Desjardins, B. (1997) Regularity Results for Two-Dimensional Flows of Multiphase Viscous Fluids. Archive for Rational Mechanics and Analysis, 137, 135-158. https://doi.org/10.1007/s002050050025

[23] Lü, B.Q. and Huang, B. (2015) On Strong Solutions to the Cauchy Problem of the Two-Dimensional Compressible Magnetohydrodynamic Equations with Vacuum. Nonlinearity, 28, 509-530. https://doi.org/10.1088/0951-7715/28/2/509

[24] Beal, J.T., Kato, T. and Majda, A. (1984) Remarks on the Breakdown of Smooth Solutions for the 3D Euler Equations. Communications in Mathematical Physics, 94, 61-66. https://doi.org/10.1007/BF01212349

[25] Liu, S.Q. and Zhang, J.W. (2016) Global Well-Posedeness for the Two-Dimensional Equations of Nonhomogeneous Incompressible Liquid Crystal Flows with Nonnegative Density. Discrete \& Continuous Dynamical Systems- B, 21, 2631-2648. https://doi.org/10.3934/dcdsb.2016065

[26] Liu, Y. and Wang, W. (2018) Strong Solutions to the Cauchy Problem of Two-Dimensional Incompressible Fluid Models of Korteweg Type. Journal of Mathematical Analysis and Applications, 465, 1075-1093. https://doi.org/10.1016/j.jmaa.2018.05.054

[27] Lions, P.L. (1996) Mathematical Topicas in Fluid Mechanics, Vol. I: Incompressible Models. Oxford University Press, Oxford.

[28] Stein, E.M. (1993) Harmonic Analysis:Real-Variable Methods, Orthogonality, and Oscillatory Integrals. Princeton University Press, Princeton.

[29] Coifman, R., Lions, P.L., Meyer, Y. and Semmes, S. (1993) Compensated Compactness and Hardy Spaces. Journal de Mathématiques Pures et Appliquées, 72, 247-286.

[30] Temam, R. (2001) Navier-Stokes Equations: Theory and Numerical Analysis. AMS Chelsea Publishing, New York. https://doi.org/10.1090/chel/343

[31] Huang, X.D., Li, J. and Xin, Z.P. (2012) Global Well-Posedness of Classical Solutions with Large Oscillations and Vacuum to the Three-Dimensional Isentropic Compressible Navier-Stokes Equations. Communications on Pure and Applied Mathematics, 65, 549-585. https://doi.org/10.1002/cpa.21382 